NASA Contractor Report 181799

ICASE REPORT NO. $89-9$

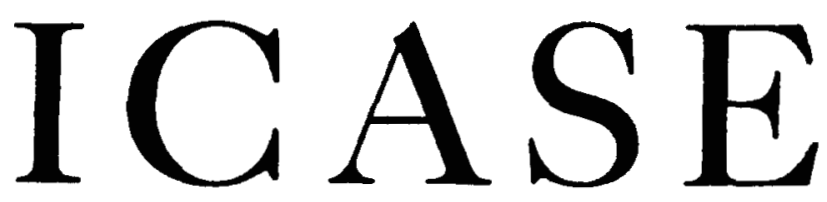

RESOLUTION REQUIREMENTS FOR NUMERICAL

SIMULATIONS OF TRANSITION

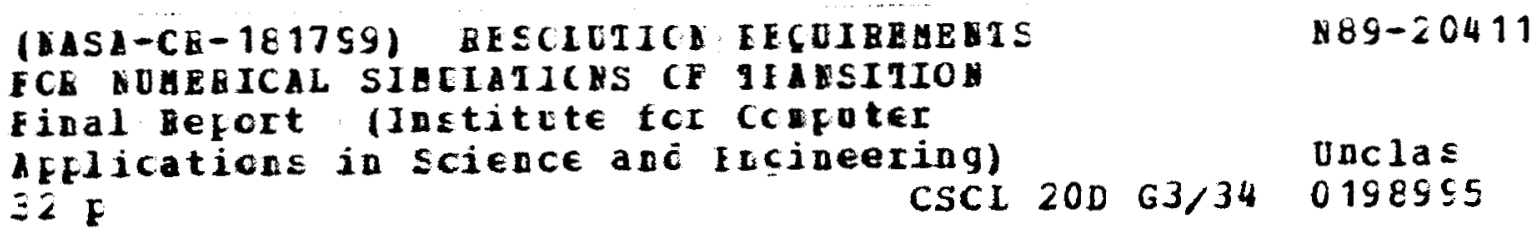

Thonas A. Zang

Steven E. Rrist

M. Yousuff Hussaini

Contract No. NAS1-18107

January 1989

INSTITUTE FOR COMPUTER APPLICATIONS IN SCIENCE AND ENGINEERING

NASA Langley Research Center, Hampton, Virginia 23665

Operated by the Universities Space Research Association

National Aeronautics and

Space Administration

Lencley Research Center

Hampton, Virginia 23665 


\title{
RESOLUTION REQUIREMENTS FOR NUMERICAL SIMULATIONS OF TRANSITION
}

Thomas A. Zang and Steven E. Krist

NASA Langley Research Center

and

M. Yousuff Hussaini ${ }^{1}$

ICASE, NASA Langley Research Center

\begin{abstract}
The resolution requirements for direct numerical simulations of transition to turbulence are investigated. A reliable resolution criterion is determined from the results of several detailed simulations of channel and boundary-layer transition.
\end{abstract}

\footnotetext{
${ }^{1}$ This research was supported by the National Aeronautics and Space Administration under NASA Contract No. NASI-18107 while the authors were in residence at ICASE, NASA Langley Research Center, Hampton, VA 23665.
} 


\title{
RESOLUTION REQUIREMENTS FOR NUMERICAL SIMULATIONS OF TRANSITION
}

\author{
Thomas A. Zang and Steven E. Krist \\ NASA Langley Research Center \\ and \\ M. Yousuff Hussaini \\ I.C.A.S.E., NASA Langley Research Center
}

\section{Introduction}

In a numerical simulation of fluid dynamics (as in an experiment) it is surely far less difficult to report what is observed than it is to judge the extent to which the observations are meaningful. Most scientists have been inculcated with a healthy skepticism about the precision of experimental data, but perhaps because of their comparative novelty (and, yes, perhaps also because of the increasingly seductive allure of computer graphics) numerical results receive less critical acceptance.

The conventional wisdom on the failings of coarse-grid numerical simulations is that artificial viscosity produces excessive smearing of the flow field. However, in certain circumstances the real flaw of coarse-grid computations is just the opposite - inadequate numerical resolution induces spurious oscillations in the flow. In direct numerical simulations of transition from laminar to turbulent flow, this latter effect is the principal one.

Transition problems can be divided into two broad categories: bifurcation problems, in which a regular sequence of instabilities arises as the Reynolds number is varied, until at large enough Reynolds number the flow is chaotic; and abrupt transition problems, in which a flow which is unstable at a fixed Reynolds number passes through a rapid sequence of instabilities leading inevitably to turbulence. Three-dimensional, nonlinear numerical calculations of the time-dependent Navier-Stokes equations have been used extensively in the last 5 to 10 years to investigate many important features of transition from laminar to turbulent flow. Taylor-Couette (Marcus (1984); Streett and Hussaini (1987)) and RayleighBenard (Curry et al (1984)) flow have been the focal points of numerical simulations of bifurcation problems, whereas free shear layers (Metcalfe et al (1987); Lowery et al (1987)) and channel and boundary-layer flows (see the review by Zang and Hussaini (1987)) have been the prime targets of abrupt transition simulations.

Orszag and Kells (1980) have characterized the artificial oscillations that arise in poorly resolved flows as "numerical turbulence". In bifurcation problems numerical turbulence 
may lead one to conclude erroneously that a given flow is chaotic (Curry et al (1984)). In abrupt transition problems, it could lead one to mislocate the instant and region in which turbulence originates as well as to mis-identify the precise instability which induces the turbulence.

Consequently, numerical simulations must be conducted with extreme care, with special attention paid to providing sufficient numerical resolution, i.e., enough grid-points. The resolution requirements for abrupt transition problems are particularly stringent, since as an unstable flow evolves towards a turbulent state the requisite resolution demands increase dramatically. In this paper we illustrate this point in some detail for some representative simulations of transition to turbulence in channel and boundary-layer flow. Moreover, we furnish some guidance on the level of accuracy that is necessary to obtain meaningful results.

\section{Numerical Approximation}

The channel flow geometry is illustrated in Figure 1. Lengths are scaled by the channel half-width $h$, velocities by the centerline velocity $u_{c}$, and the density by its constant value. The Reynolds number $R e$ is based on these scales, i.e., $R e=u_{c} h / \nu$, where $\nu$ is the kinematic viscosity. The flow is assumed to be fully developed with the parabolic mean flow profile $u_{0}(y)=1-y^{2}$.

In dimensionless variables, the incompressible Navier-Stokes equations in rotation form may be written

$$
\begin{gathered}
\frac{\partial \mathbf{u}}{\partial t}+\omega \times \mathbf{u}+\nabla\left(\frac{1}{2}|\mathbf{u}|^{2}\right)+\nabla p=\nu \Delta \mathbf{u} . \\
\nabla \cdot \mathbf{u}=0
\end{gathered}
$$

where $\mathbf{u}=(u, v, w)$ is the velocity, $\boldsymbol{\omega}=\boldsymbol{\nabla} \times \mathbf{u}$ is the vorticity, and $p$ is the pressure. The independent variables are assumed to be periodic in $x$ and $z$, with no-slip conditions on $\mathbf{u}$ at the walls $y= \pm 1$.

Two related numerical methods have been used to obtain the results reported herein. Both use a Fourier collocation technique for the spatial discretization in the $x$ and $z$ directions. One uses a second-order finite-difference scheme in $y$, and the other uses Chebyshev collocation for that direction. A Fourier-finite-difference method (FFDM) was devised by Wray and Hussaini (1984) for direct simulation of boundary-layer transition and by Moin and Kim (1982) for large-eddy simulation of channel flow; the latter version was used by Biringen $(1984,1987)$ for his simulations of channel flow transition and for some of the present results. The particular Fourier-Chebyshev method (FCBM) used here is the one developed by Zang and Hussaini (1986). The FCBM code uses the standard Chebyshev grid in $y$,

$$
y=-\cos \xi,
$$


with the grid-points uniformly distributed in the computational variable $\xi \epsilon[0, \pi]$. The FFDM code uses the hyperbolic tangent transformation,

$$
y=\frac{1}{a} \tanh \left(\xi \tanh ^{-1} a\right)
$$

with the stretching parameter $a=0.94175$ and $\xi \epsilon[-1,1]$. Both methods employ an explicit time discretization for the advection and horizontal diffusion terms and an implicit time discretization for the normal diffusion and the pressure gradient terms as well as for the continuity equation. The FCBM uses a splitting, or fractional-step, technique for the time advancement: in the first half-step the advection and diffusion terms are accounted for, and in the second half-step a pressure correction is applied so that the final velocity field is divergence free. Boundary conditions are applied at the intermediate step to all three velocity components and in the pressure correction to the normal velocity component. The FFDM is unsplit, i.e., all terms are accounted for simultaneously in a single step. Both codes employ a staggered grid for the pressure so that no artificial boundary conditions are required. A thorough discussion of the available spectral algorithms for channel flow simulations is provided in Chapter 7 of Canuto, et al (1988).

Recently, Zang (1989) demonstrated that in numerical simulations utilizing collocation, it is much better to use the Navier-Stokes equations in the skew-symmetric form, given by

$$
\frac{\partial \mathrm{u}}{\partial t}+\frac{1}{2} \mathrm{u} \cdot \nabla \mathbf{u}+\frac{1}{2} \nabla \cdot(\mathbf{u u})+\nabla p=\nu \Delta \mathbf{u}
$$

than in the commonly used rotation form given by Eq. (1). Several numerical examples were presented, including one for channel flow, which demonstrated the superiority of the skew-symmetric form. This result is quite recent and post-dates some of the calculations reported in the present paper. Unless otherwise noted, the computations reported here utilized the skew-symmetric form.

In these algorithms, both physical space and spectral space representations of the dependent variables are employed. The velocity has the Fourier series representation

$$
\mathrm{u}(\mathrm{x}, t)=\mathrm{u}_{0}(y)+\sum_{k_{z}=-N_{z} / 2}^{N_{z} / 2-1} \sum_{k_{x}=-N_{z} / 2}^{N_{z} / 2-1} \hat{\mathbf{u}}_{k_{x}, k_{z}}(y, t) e^{\left[k_{z} \alpha x+k_{x} \beta z\right]},
$$

where $\mathbf{u}(y)=\left(u_{0}(y), 0,0\right)$, and $\alpha$ and $\beta$ are the fundamental wavenumbers in the streamwise and spanwise directions respectively. The fundamental wavelengths in these directions are given by $L_{x}=2 \pi / \alpha$ and $L_{z}=2 \pi / \beta$, respectively. The imposed periodicity lengths are $L_{x}$ and $L_{z}$. The integers $k_{x}$ and $k_{z}$ label the Fourier wavenumbers in the numerical representation with respect to the fundamental wavenumbers $\alpha$ and $\beta$. The velocity also has the Chebyshev series representation

$$
\mathbf{u}(\mathbf{x}, t)=\sum_{n=0}^{N_{\mathbf{y}}} \tilde{\mathbf{u}}_{n}(x, z, t) T_{n}(y) .
$$


For later reference we list here a useful measure of the strength of a given Fourier harmonic:

$$
E_{k_{x}, k_{x}}(t)=d_{k_{z}} d_{k_{s}} \int_{-1}^{1}\left|\hat{\mathbf{u}}_{k_{x}, k_{x}}(y, t)\right|^{2} d y / E_{0}
$$

where

$$
d_{k}=2-\delta_{k 0} .
$$

This quantity is the kinetic energy of the mode normalized by the kinetic energy of the mean flow $\left(E_{0}\right)$. The constant $d_{k}$ is used to account for the symmetries in the Fourier harmonics. Similarly, a measure of a given Chebyshev component is

$$
E_{n}(t)=\int_{0}^{L_{z}} \int_{0}^{L_{z}}\left|\tilde{\mathbf{u}}_{n}(x, z, t)\right|^{2} d x d z /\left(L_{x} L_{z} E_{0}\right) .
$$

Note that $E_{0,0}(t)$ and $E_{0}(t)$ measure the kinetic energy of the mean flow correction (see Eq. (6)).

The initial conditions for the transition simulations reported in this work have the form

$$
\mathbf{u}(\mathbf{x}, 0)=\Re e\left\{\mathbf{u}_{0}(y)+\epsilon_{2 D} e^{i \theta} \mathbf{u}_{2 D}(y) e^{i \alpha x}+\frac{1}{2} \epsilon_{3 D}\left[\mathbf{u}_{3 D}^{+}(y) e^{i(\alpha x+\beta x)}+\mathbf{u}_{3 D}^{-}(y) e^{i(-\alpha x+\beta z)}\right]\right\},
$$

where $\mathbf{u}_{2 D}(y)$ and $\mathbf{u}_{3 D}^{ \pm}(y)$ are the least stable linear modes for the given real wavenumbers $\alpha$ and $\beta$. These eigenfunctions are commonly normalized so that their maximum streamwise amplitudes are 1, and so that, when used in the form given in Eq. (11), these maxima occur at $x=0$ and $z=0$ for $\theta=0$. Thus, $\epsilon_{2 D}$ and $\epsilon_{3 D}$ measure the maxima of the streamwise fluctuations of the 2-D and oblique disturbances relative to the mean centerline velocity, and $\theta$ measures the phase shift between the 2-D and the oblique waves.

\section{$\underline{A} \mathbf{R e}=1500$ Channel Transition}

The particular channel flow transition studied by Biringen $(1984,1987)$ is used here as the primary example of the resolution demands of transition simulations. The Reynolds number is 1500 . The horizontal wave numbers $\alpha$ and $\beta$ are both unity. The temporal frequencies of the least rapidly damped modes are $\omega_{2 D}=0.326299-0.028206 i$ and $\omega_{3 D}=0.401293-0.028230 i$, as determined by a Chebyshev tau approximation to the OrrSommerfeld equation (Orszag (1971)). The initial amplitudes of the waves were $\epsilon_{2 D}=0.11$ and $\epsilon_{3 D}=0.10$, and the phase shift was $\theta=-37^{\circ}$. The numerical computations are made in a frame moving with a streamwise velocity of 0.36 . This is the average of the phase speeds of the two- and three-dimensional waves. The use of a moving frame improves the temporal accuracy of the calculation. All calculations presented in this section employed the rotation form.

A straightforward test of the resolution in the normal direction is the ability of a code to reproduce the time evolution of a linear eigenfunction. The computed growth rates and their errors at $t=\mathbf{3 0}$ are given in Table $\mathbf{1}$ for several calculations. The initial condition 
for each case consisted of the mean flow plus an oblique TS wave at $0.01 \%$ amplitude. Since Fourier collocation is used in the horizontal directions and the linear solution is a pure trigonometric function in $x$ and $z$, a mere 4 points in each of these directions suffices for essentially perfect resolution. The time-step was chosen sufficiently small so that the errors were dominated by the normal discretization. The Chebyshev calculation is accurate to better than five significant digits. The finite-difference calculations exhibit the expected second-order accuracy. Moreover, the finite-difference calculations err on the side of excessive damping of the wave. (This same effect was found by Malik, Zang, and Hussaini (1985) at a higher Reynolds number.)

\begin{tabular}{|c|c|r|c|}
\hline Algorithm & $N_{v}$ & Calculated $\omega_{i}$ & Error in $\omega_{i}$ \\
\hline Exact & & -0.028230 & \\
\hline FCBM & 64 & -0.028226 & -0.000004 \\
\hline FFDM & 50 & -0.032030 & -0.003800 \\
\hline FFDM & 100 & -0.029230 & -0.001000 \\
\hline FFDM & 200 & -0.028482 & -0.000252 \\
\hline
\end{tabular}

Table 1: Effect of Normal Resolution on Growth Rate

The error in the $N_{y}=50$ FFDM growth rate might appear to be small; after all, it is less than $1 \%$ when normalized by $\left|\omega_{3 D}\right|$. Nevertheless, it portends uncomfortably large errors in the full transition simulation. These manifest themselves quite clearly in the timedependent vortical flow field. Since the vorticity is obtained from the primitive variables by differentiation, it is a sensitive indicator of errors in the velocities themselves.

At early stages in the transition process there are two pronounced structures in the flow: a predominantly streamwise vortex known as a lambda vortex and a strong detached shear layer which lies above it. These features have been documented experimentally by Williams, Fasel, and Hama (1984) and numerically by Krist and Zang (1987), among others. The bottom row of Figure 2 shows the results in the lower half of the channel from a well-resolved, $64^{3}$ FCBM calculation at $t=15$. The two quantities displayed are the vertical shear, $\partial u / \partial y$, and the streamwise vorticity, $\omega_{x}$. The former is shown in the spanwise plane of symmetry, $z / L_{z}=1 / 2$, which is the so-called "peak plane". The detached shear layer is located in the upper left half of the frame. The latter is given for a streamwise plane near the maximum vertical shear, in this case at $x / L_{x}=1 / 2$. The lambda vortex is reflected by the uppermost counter-rotating features near the center of the frame. An thorough description of the flow field for this case can be found in Krist and Zang (1987).

The results from a $32 \times 50 \times 32$ FFDM calculation and from a $32 \times 64 \times 32$ FCBM calculation are shown in the top two rows of Figure 2. (The FFDM grid was chosen to match that used by Biringen (1984).) The two fully spectral results are in close agreement. The 
coarser one does, however, exhibit some slight oscillations in the streamwise vorticity contours. The errors in this calculation stem primarily from inadequate spanwise resolution. Errors in the FFDM calculation are much more pronounced. The detached shear layer clearly lags behind its true position. Moreover, there are spurious streamwise vorticity regions located above the lambda vortex. The principal errors here are those due to the discretization in the normal direction; errors from the inadequate spanwise resolution are present at about the same level as in the coarse FCBM calculation. Krist and Zang (1987) compared the FFDM results shown here with FFDM results on a $32 \times 200 \times 32$ grid. The phase lag of the detached shear layer is not nearly so severe, and the spurious streamwise vorticity regions are smaller. Nevertheless the results are still markedly inferior to those from the $32 \times 64 \times 32$ FCBM. Bear in mind that Table 1 indicates that with 200 normal grid-points, the error of the FFDM on the primary waves is less than $0.1 \%$. This fact underscores the extreme resolution demands posed by numerical simulations of transition to turbulence.

After $t=15$, the flow field evolves rapidly and the resolution demands increase substantially. Figure 3 presents the results for the coarse-grid FFDM, the $64^{3}$ FCBM, and a well-resolved FCBM at $t=18.75$. (The well-resolved results here and in Figures 6 and 7 were the product of a single simulation in which the grid was refined (using spectral interpolation) whenever further resolution was required.) Note that the detached shear layer has intensified as has the streamwise vorticity. Notice also that the streamwise vortex has pinched closer towards the peak plane and that it has narrowed its dimensions in the spanwise direction. Hence, the flow field contains sharper gradients and more grid-points are needed. In fact, the spanwise resolution demands are more than twice as great as they were at $\mathrm{t}=15$. The $64^{3} \mathrm{FCBM}$ result is plainly inadequate. The streamwise vorticity contains an obvious grid-scale oscillation, and problems are evident in the vertical shear near $x / L_{x}=3 / 4$. The $32 \times 50 \times 32 \mathrm{FFDM}$ result is afflicted with severe oscillations in the streamwise vorticity, and the quality of the vertical shear has also deteriorated. The effects of the phase lag are still present.

The Fourier-Chebyshev coefficients of the computed solution are a good indicator of the accuracy of a fully spectral result. The resolution spectra of several cases are shown in Figure 4. The resolution in the normal direction is measured by the quantity $E_{n}(t)$ defined by Eq. (8), whereas the resolutions in $x$ and $z$ are measured by

$$
E_{k_{z}}(t)=\max _{k_{z}} E_{k_{x}, k_{x}}(t)
$$

and

$$
E_{k_{z}}(t)=\max _{k_{z}} E_{k_{z}, k_{z}}(t),
$$

respectively. Consider first the $64^{3}$ case at $t=15$. There is a decay of at least 8 orders of magnitude from the low wavenumber end to the high wavenumber end of the resolution spectra in each coordinate direction. Since the spectra are quadratic in the perturbation velocities, this corresponds to a truncation error of better than $0.01 \%$ in the primitive variables themselves. The resolution spectra in $x$ and $z$ have a mild curl at their tails. In the case of the $x$-direction this is due to the round-off error of these 32-bit calculations. 
The curl of the tail in the $z$-direction is at a higher level than can be accounted for by round-off error. It signals an imminent loss of resolution on this grid, a claim supported by the $64^{3}$ results at $t=18.75$ shown in Figure 3 . The resolution spectra at $t=18.75$ for the well-resolved case also exhibit an 8 order of magnitude decay. In neither the $t=15$, $64^{3}$ nor the $t=18.75,96 \times 128 \times 162$ contour plots is there visible evidence of oscillations. Thus, in both of these well-resolved cases the lack of visible evidence of oscillations in the vorticity field corresponds to a truncation error of less than $0.01 \%$.

The bottom right example in Figure 4 is for the under-resolved FCBM case at $t=$ 18.75. The resolution spectra suggest that the $x$-direction is resolved the best, and the $z$-direction the worst. This is consistent with the middle row of Figure 3, which indicates severe oscillations in the spanwise direction and few problems in the streamwise direction.

One must be careful in how the data is plotted when looking for small-scale oscillations. This point is driven home by Figure 5. Here the results of a $64^{3}$ FFDM simulation at $t$ $=18.75$ are plotted in two ways. In the top row, all points in the flow field are used in the plotting. In the bottom row, every other point (in the horizontal directions) has been discarded before plotting. Notice that the flow field appears to be much smoother in the bottom case. The explanation is that the most severe oscillations are of the twopoint, sawtooth variety and these are exactly eliminated by the coarsening procedure. Since simulations of transition require large data bases, this type of coarsening for data reduction may be adopted in the interests of conserving storage. Nevertheless, it can present a very misleading picture of the reliability of the results. In addition, the use of plotting routines which deliberately smooth the data should be avoided.

Incidentally, a comparison of the top row of Figure 5 with the top two rows of Figure 3 confirms that the spurious streamwise vorticity regions are indeed due to the FFDM's inadequate normal resolution.

The effects of inadequate resolution are even more pronounced at later times. Figure 6 shows the computed flow at $t=22.5$. The FFDM results exhibit only the barest resemblance to even the large-scale vertical shear, and the streamwise vorticity is extremely corrupted. The coarse-grid FCBM flow contains large-amplitude grid-scale oscillations in the streamwise vorticity, but the vertical shear is still reasonable. The resolution spectra for the well-resolved case is given in the lower left frame of Figure 4. Once again the 8 order of magnitude decay correlates well with the absence of visible flow-field oscillations.

The $t=27.5$ results are given in Figure 7. The instantaneous vorticity field from the FFDM is now completely unreliable. The large-scale features of the coarse-grid FCBM vertical shear are still correct. However, there is substantial contamination of the smallscale features in the bottom quarter of the plot, near the lower wall of the channel. The well-resolved case still has a smooth flow field and its resolution spectra (not shown) are again indicative of a $0.01 \%$ truncation error.

From $t=25$ onwards the flow is undergoing the final stages of transition to turbulence. As documented by Zang and Krist (1989), the principal flow field activity shifts markedly downward towards the near-wall region. It is precisely here that the coarse-grid results are 
most unrealistic. Hence, they are effectively useless in addressing the physics of the final stages of transition.

The preceding comparisons indicate that loss of resolution on the small-scale flow features leads eventually to loss of resolution on the large-scale features. Quantities such as the resolution spectra appear to be the best measure of the resolution for spectral methods. Unfortunately, the resolution spectra are not quite as meaningful in a direction which is discretized via finite differences, since the spectra are not so directly related to the numerical approximation.

The $0.01 \%$ accuracy criterion that we advocate here is by no means definitive. We have seen, however, that even an order of magnitude relaxation in this criterion leads to obvious effects on the large-scale field. Hence, it is the least that one should require for a transition simulation.

\section{$\underline{A} \mathbf{R e}=5000$ Channel Transition}

Gilbert and Kleiser (1986) have simulated channel flow transition at $\mathrm{Re}=5000$. Their calculation was performed on a $64 \times 128 \times 128$ grid and they were able to maintain an 8 order of magnitude reduction in the spectra until $t=134$. The results of the previous section indicate that greater resolution was required at the lower Reynolds number of 1500 . This seems counter-intuitive since one expects smaller scales to develop at the higher Reynolds number. One reason behind the need for greater resolution for the $R e=1500$ case is that the initial amplitudes are 3 times larger at the lower Reynolds number. Nonlinear effects lead to a more rapid saturation of the higher Fourier-Chebyshev harmonics. Moreover, the Chebyshev distribution of grid-points in the normal direction is better tuned to the flow field at $\operatorname{Re}=5000$, which is more concentrated towards the walls.

These conclusions are supported by our own calculation under the Gilbert and Kleiser conditions. The fundamental wavenumbers were $\alpha=1.12$ and $\beta=2.10$. The initial amplitudes were $\epsilon_{2 D}=0.03$ and $\epsilon_{3 D}=0.001$, and the initial phase shift $\theta=0^{\circ}$. They used the constant flow rate formulation rather than the constant pressure gradient formulation employed in the our $\operatorname{Re}=1500$ calculations. (As discussed by them, the flow settles down to a statistically steady turbulent state sooner under the constant flow rate condition.) In our repeat of this calculation the constant flow rate formulation was used, and it was run on a 64-bit machine. Compared with the case discussed in the previous section, this transitional flow takes a lot longer to reach a strongly nonlinear stage since it began from much smaller initial amplitudes.

Figure 8 shows the vertical shear and the streamwise vorticity at $t=128, t=134$, and $t=138$. The resolution spectra at these times are given in Figure 9. There are no visible oscillations at $t=128$ and $t=134$ and the resolution spectra exhibit the 8 order of magnitude decay that was found necessary at $R e=1500$. This criterion is not satisfied at $\mathrm{t}=138$, particularly in the $x$-direction, and the oscillations in the top right frame of Figure 8 are the consequence. We find that, strange as it may seem at first sight, it is 
easier to simulate transition at $\mathbf{R e}=\mathbf{5 0 0 0}$ than at $\mathbf{R e}=\mathbf{1 5 0 0}$.

Notice that the curl in the tails of the resolution spectra are much milder here than they were for the Re $=1500$ case. As documented by Zang (1989), this is one benefit of the skew-symmetric form of the Navier-Stokes equations.

\section{A Re $=1100$ Boundary-Layer Transition}

Recently, Zang, Hussaini, and Erlebacher have performed a high resolution simulation of boundary-layer transition under the conditions of the experiment by Kovasznay, Komoda, and Vaseduva (1962). (See Zang and Hussaini (1987) for some preliminary results.) The Reynolds number, based on displacement thickness and the free stream velocity, was 1100 , and the fundamental wavenumbers were $\alpha=0.250$ and $\beta=0.209$. The initial amplitudes were $\epsilon_{2 D}=0.018$ and $\epsilon_{3 D}=0.008$, and the initial phase shift $\theta=-36^{\circ}$. The simulation was performed under the parallel flow assumption in which the non-parallel effects are ignored. This permits much better resolution of the flow at the price of neglecting some of the physics of a true, growing boundary layer (see Zang and Hussaini (1987)).

The calculation was performed on a $144 \times 144 \times 324$ grid and Zang, Hussaini, and Erlebacher reproduced numerically the hydrogen-bubble flow visualization results of Hama and Nutant (1963). They maintained an 8 order of magnitude reduction in the spectra throughout the calculation. Figure 10, taken from that work, displays the vertical shear and the mean velocity profiles in the peak plane. The horizontal direction encompasses one period of the TS wave and the vertical direction covers 6 displacement thicknesses of the undisturbed boundary layer. The vertical shear clearly indicates the primary roll-up of the shear layer. (The times in the figure are given in units of the period of the 2-D wave.) By Frame 6 a secondary roll-up is just commencing at a height of about 2.5 displacement thicknesses.

Some questions have arisen as to how much resolution is required to reproduce the essential features of this flow, at least in the peak plane. This is a particularly important question for true spatial simulations because the limitations of current computers mandate that resolution in the spanwise direction be sacrificed to furnish the much greater resolution needed in the streamwise direction for the spatially-growing boundary layer. To date spatial simulations have employed drastic spanwise truncations, retaining only between 4 and 32 points in $z$ (see Fasel, Rist, and Konzelmann (1987)). With so few points in the spanwise direction, truly small-scale spanwise oscillations will not occur - they will be on the scale of the spanwise grid, which in these cases is quite coarse. One might hope that such simulations will still capture the essential physics at the later stages of transition.

To investigate this issue, we repeated the Zang, Hussaini and Erlebacher parallel boundary-layer simulation from $t=3$ onwards using a collocation grid of $96 \times 96 \times 12$. To increase the accuracy of this drastically truncated simulation, de-aliasing was applied in the $x$ and $z$ directions using the 2/3-rule (see Canuto et al (1988, Chapter 3)). The results are shown in Figure 11. The contrast with the well-resolved flow is substantial. 
The detached shear layer is much less intense, it stays much lower in the boundary layer, it lags behind its true streamwise position, and its roll-up is quite mild.

These plots permit some inferences about what part of the physics of transition is mis-represented by the coarse-grid simulation. A principal effect in this type of transition (see Zang and Krist (1989)) is the pinching of the lambda vortex towards the peak plane and its lifting away from the wall and towards the free stream. Much of this behavior is due to the self-induction of the lambda vortex. The intensity of the self-induction is tied to the strength of the vorticity. On a coarse grid, only a relatively weak lambda vortex is supportable. Hence, the evolution of the lambda vortex is retarded, both in the rate at which it pulls away from the wall and the rate at which it moves in the downstream direction. Since the detached shear layer is tied to the top of the lambda vortex, its intensity, location, and speed is affected in like manner. Figures 10 and 11 are but a dramatic illustration of this effect.

In this simulation even though the resolution in $z$ was woefully inadequate, the resolution in $x$ and $y$ was kept sufficient to maintain $0.01 \%$ accuracy in those directions. The fact that fewer grid-points were required here reflects the phenomenon that since only large scales are supported in $z$ and the scales in the three coordinate directions are inter-related, fewer small-scale features appear in the other directions. The unfortunate fact is that the more grid-points that are used in the spanwise direction, the more that are also needed in the other two directions.

These two simulations beg the question of just how much spanwise resolution is enough. Figures 12-14, which contain the results of simulations employing 18, 24, and 48 spanwise grid-points, respectively, provide further information. The results clearly improve, i.e., tend more towards the well-resolved case, as the number of spanwise grid-points increases. All of these intermediate cases exhibit the primary roll-up of the shear layer. However, these also display a premature secondary roll-up.

The principal flaw in them appears to be the occurrence of regions of intense reverse spanwise vorticity. The mean wall shear is 0.57 , the peak value in the detached shear layer of the well-resolved case at Frame 6 is 2.4, and the minimum value is $\mathbf{- 0 . 2}$. The color bar was cut off at $\partial u / \partial y<-0.5$. Values less than -0.5 appear as white regions. These occur below and just upstream of the secondary roll-up in the shear layer. They are as strong as $-1.0,-1.5$, and -1.5 in the respective Frame 6 portions of Figures 12, 13, and 14 . (Although these figures display the vertical shear, they correspond closely to the (negative of the) spanwise vorticity.) Thus, all the intermediate cases are corrupted by the presense of incorrect regions of reversed spanwise vorticity. In the case $N_{s}=48$ these is additional corruption in the near wall region. This reversed vorticity has as yet undetermined effects on the physics of the flow but appears related to an exaggeration of the shear-layer roll-up.

\section{Discussion}

The emphasis in this paper has been on inferring what degree of resolution is needed 
to capture reliably the instantaneous structure of a transitional flow. This requirement stems from the desire to use numerical simulations to clarify the details of the final stages of transition and to assist in the development of reliable models for this important flow regime. These are more challenging goals numerically than simulating the low-order statistics of a turbulent steady state. Direct numerical simulations of turbulent channel flow have typically settled for a 3 or 4 order of magnitude reduction in the one-dimensional energy spectra (see, for example, Kim, Moin, and Moser(1987)). Turbulence simulations have even produced reasonable mean flow and velocity fluctuation profiles in channel flow on an $8 \times 32 \times 8$ grid (Gilbert and Kleiser (1986)). These results, however, were obtained with the assistance of a temporal average of the computed results over a substantial time interval.

This option is not available for transition simulations. Some alternatives to the straightforward simulations presented here are the incorporation of suitable models of subgrid-scale transition structures and the development of efficient self-adaptive numerical algorithms that automatically concentrate the grid-points in regions of small-scale structure. Neither of these developments, however, is an easy task.

\section{Conclusion}

Viable short-cuts for reliable direct simulations of transition to turbulence are hard to come by.

\section{References}

Biringen, S. (1984): Final stages of transition to turbulence in plane channel flow, J. Fl. Mech., Vol. 148, pp. 413-442.

Biringen, S. (1987): Three-dimensional Vortical Structures of Transition in Plane Channel Flow, AIAA Paper No. 87-0046.

Canuto, C.; Hussaini, M. Y.; Quarteroni, A.; and Zang, T. A. (1988): Spectral Methods in Fluid Dynamics, Springer-Verlag, Berlin.

Curry, J.; Herring, J.; Loncaric, J.; and Orszag, S. A. (1984): Order and disorder in twoand three-dimensional Benard convection, J. Fl. Mech., Vol. 147, pp. 1-38.

Fasel, H.; Rist, U.; and Konzelmann, U. (1987): Numerical Investigation of the ThreeDimensional Development in Boundary Layer Transition, AIAA Paper No. 87-1203.

Gilbert, N.; and Kleiser, L. (1986): Subcritical transition to turbulence in channel flow, in Direct and Large Eddy Simulation of Turbulence, U. Schumann and R. Friedrich (eds.), pp. 1-18, Vieweg, Braunschweig. 
Hama, F. R.; and Nutant, J. (1963): Detailed flow-field observations in the transition process in a thick boundary layer, in Proc. of the 1963 Heat Transfer and Fluid Mechanics Institute, pp. 77-93, Stanford Univ. Press, Palo Alto.

Kim, J.; Moin, P.; and Moser, R. (1987): Turbulence statistics in fully developed channel flow at low Reynolds number, J. Fl. Mech., Vol. 177, pp. 133-166.

Kovasznay, L. S.; Komoda, H.; and Vasudeva, B. R. (1962): Detailed flow field in transition, in Proc. 1962 Heat Transfer and Fluid Mechanics Institute, pp. 1-16, Stanford Univ. Press, Palo Alto.

Krist, S. E.; and Zang, T. A. (1987): Numerical Simulation of Channel Flow Transition, NASA TP 2667.

Lowery, P. S.; Reynolds, W. C.; and Mansour, N. N. (1987): Passive Scalar Entrainment and Mixing in a Forced, Spatially-developing Mixing Layer, AIAA Paper No. 870132 .

Malik, M. R.; Zang, T. A.; and Hussaini, M. Y. (1985): A spectral collocation method for the Navier-Stokes equations, J. Comput. Phys., Vol. 61, pp. 64-88.

Marcus, P. S. (1984): Simulation of Taylor-Couette flow. Part 2. Numerical results for wavy vortex flow with one traveling wave, J. Fl. Mech., Vol. 146, pp. 65-113.

Metcalfe, R. W.; Orszag, S. A.; Brachet, M. E.; Menon, S.; and Riley, J. J. (1987): Secondary instability of a temporally growing mixing layer, J. Fl. Mech., Vol. 184, pp. 207-243.

Orszag, S. A. (1971): Accurate solution of the Orr-Sommerfeld equation, J. Fl. Mech., Vo. 50, pp. 689-703.

Orszag, S. A.; and Kells, L. C. (1980): Transition to turbulence in plane Poiseuille flow and plane Couette flow, J. Fl. Mech., Vol. 96, pp. 159-205.

Streett, C. L.; and Hussaini, M. Y. (1987): A Numerical Simulation of Finite-length Taylor-Couette Flow, AIAA Paper No. 87-1444.

Williams, D. R.; Fasel, H.; and Hama, F. R. (1984): Experimental determination of the three-dimensional vorticity field in the boundary layer transition process, J. Fl. Mech., Vol. 149, pp. 179-203.

Wray, A. A.; and Hussaini, M. Y. (1984): Numerical experiments in boundary-layer stability, Proc. Roy. Soc. London, Ser. A, Vol. 392, pp. 373-389.

Zang, T. A. (1989): On the rotation and skew-symmetric forms for incompressible flow simulations, Appl. Numer. Math., in press.

Zang, T. A.; and Hussaini, M. Y. (1986): On spectral multigrid methods for the timedependent Navier-Stokes equations, Appl. Math. Comp., Vol. 19, pp. 359-372. 
Zang, T. A.; and Hussaini, M. Y. (1987): Numerical simulation of nonlinear interactions in channel and boundary-layer transition, in Nonlinear Wave Interactions in Fluids AMD-Vol. 87, R. W. Miksad, T. R. Akylas and T. Herbert, eds., ASME, New York, pp. 131-145.

Zang, T. A.; and Krist, S. E. (1989): Numerical experiments on stability and transition in plane channel flow, Theor. and Comput. Fl. Dyn., Vol. 1, in press. 


\section{Captions}

Figure 1. Schematic of the channel flow problem. The streamwise coordinate is denoted by $x$, the coordinate normal to the walls by $y$, and the spanwise coordinate by $z$.

Figure 2. Vertical shear (left) and streamwise vorticity (right) contours in the lower half of the $\operatorname{Re}=1500$ channel at $t=15$. The contour interval is 0.40 . Dashed lines indicate negative contours.

Figure 3. Vertical shear (left) and streamwise vorticity (right) contours in the lower half of the $R e=1500$ channel at $t=18.75$.

Figure 4. Resolution spectra in each of the coordinate directions for $\mathrm{Re}=1500$ channel flow simulations.

Figure 5. Vertical shear (left) and streamwise vorticity (right) contours in the lower half of the $R e=1500$ channel at $t=18.75$ for a $64^{3}$ FFDM simulation.

Figure 6. Vertical shear (left) and streamwise vorticity (right) contours in the lower half of the $R e=1500$ channel at $t=22.5$.

Figure 7. Vertical shear (left) and streamwise vorticity (right) contours in the lower half of the $\operatorname{Re}=1500$ channel at $t=27.5$.

Figure 8. Vertical shear (top) and streamwise vorticity (bottom) contours in the lower half of the $\mathbf{R e}=\mathbf{5 0 0 0}$ channel. The contour interval is $\mathbf{0 . 4 0}$.

Figure 9. Resolution spectra in each of the coordinate directions for the $\operatorname{Re}=\mathbf{5 0 0 0}$ channel flow simulation.

Figure 10. Vertical shear contours and mean velocity profiles for the $R e=1100$ boundarylayer simulation on a $144 \times 144 \times 324$ grid.

Figure 11. Vertical shear contours and mean velocity profiles for the $R e=1100$ boundarylayer simulation on a $96 \times 96 \times 12$ grid.

Figure 12. Vertical shear contours and mean velocity profiles for the $R e=1100$ boundarylayer simulation on a $192 \times 128 \times 18$ grid.

Figure 13. Vertical shear contours and mean velocity profiles for the $\operatorname{Re}=1100$ boundarylayer simulation on a $192 \times 96 \times 24$ grid.

Figure 14. Vertical shear contours and mean velocity profiles for the $R e=1100$ boundarylayer simulation on a $256 \times 128 \times 48$ grid. 


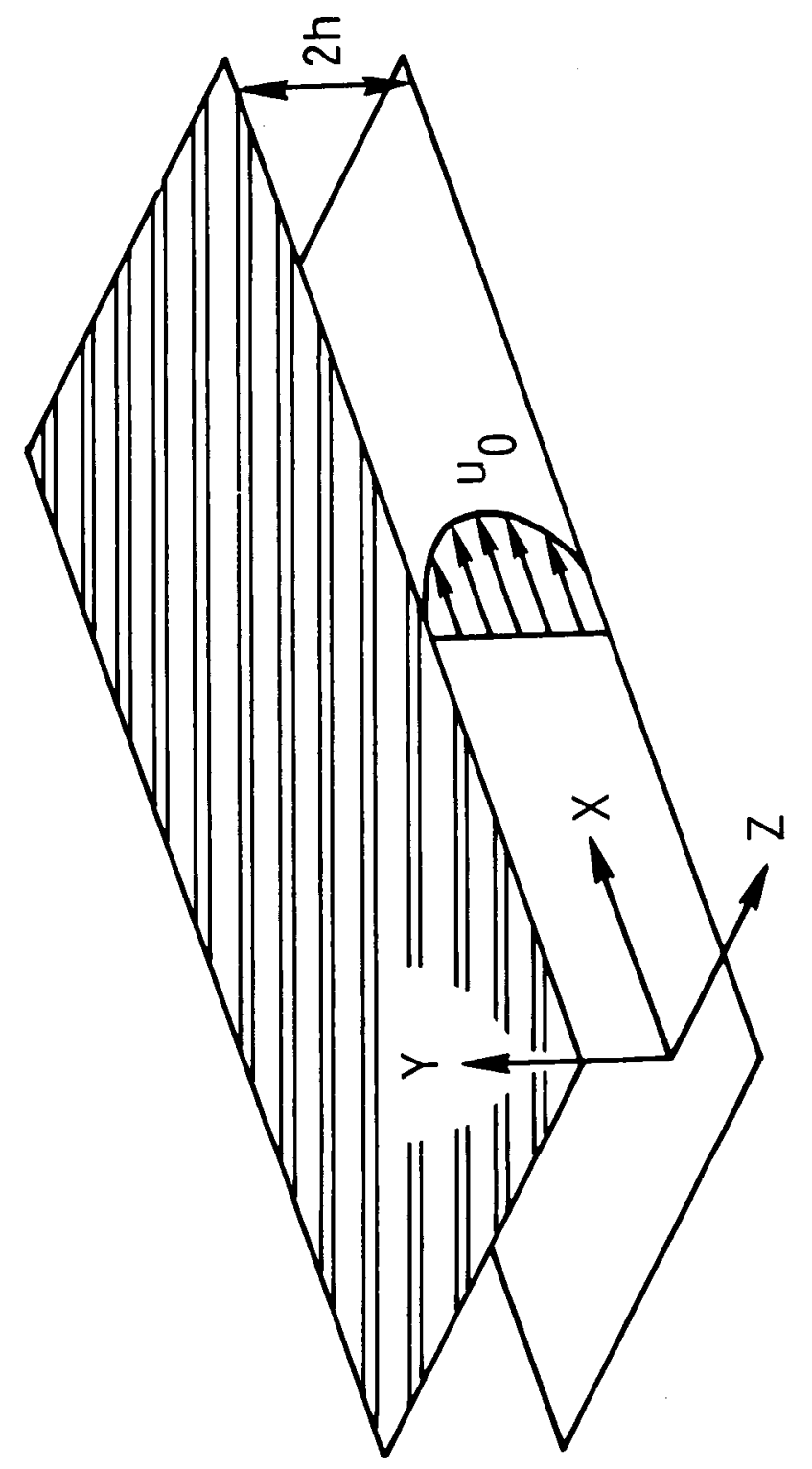

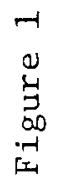


32×50x32 FFDM
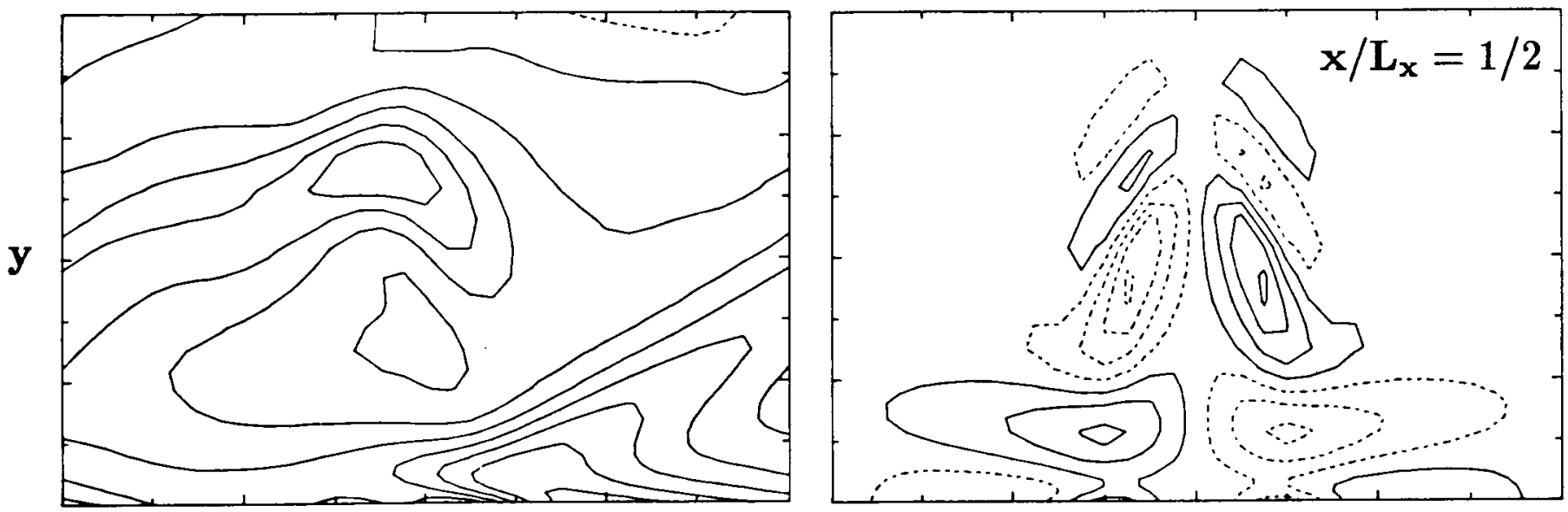

$32 \times 64 \times 32$ FCBM
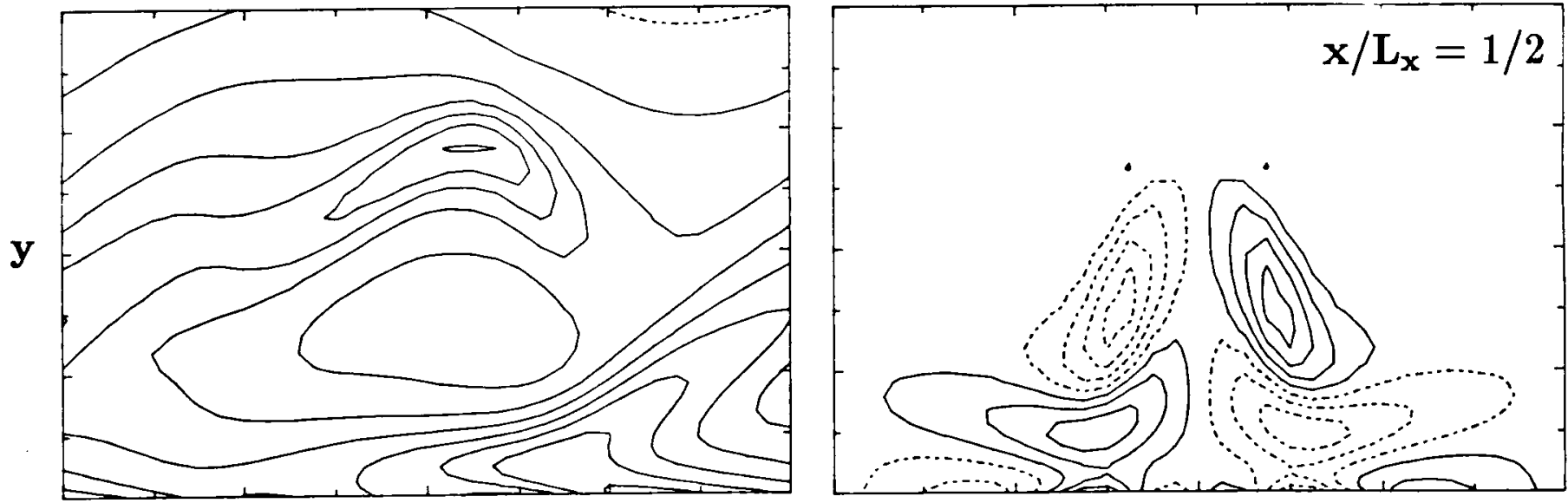

64x64x64 FCBM
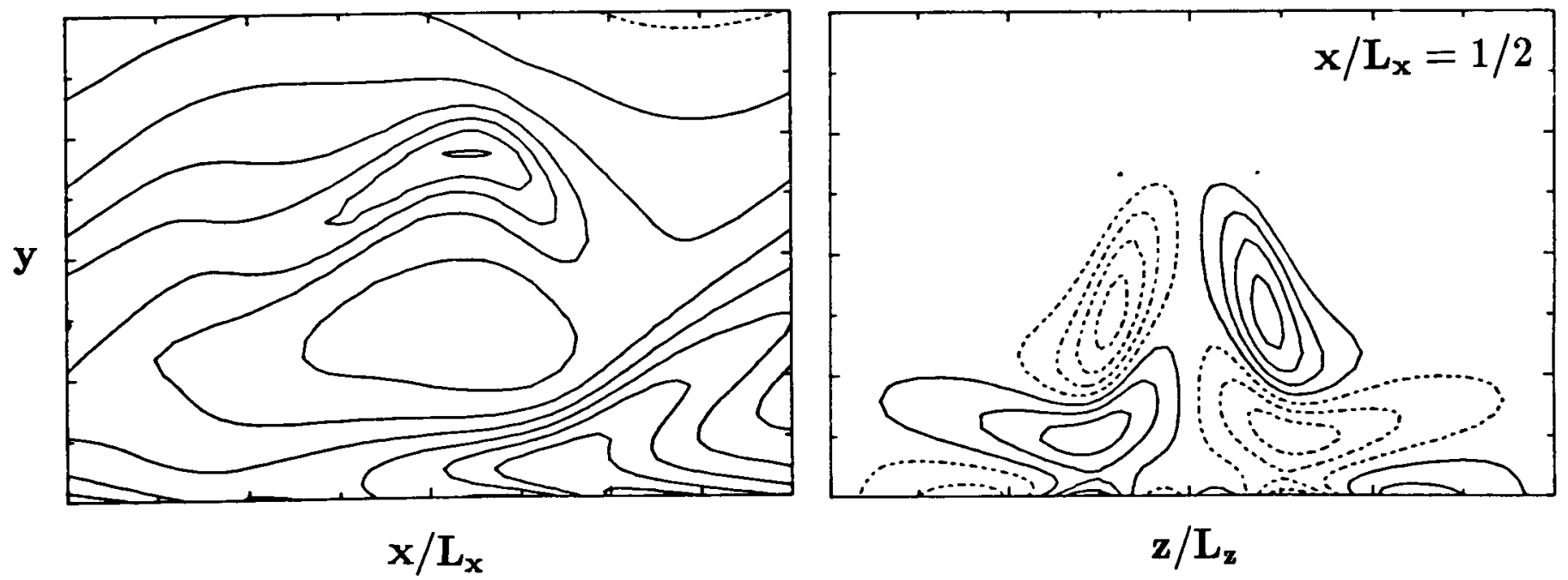

Figure 2 
32x50x32 FFDM
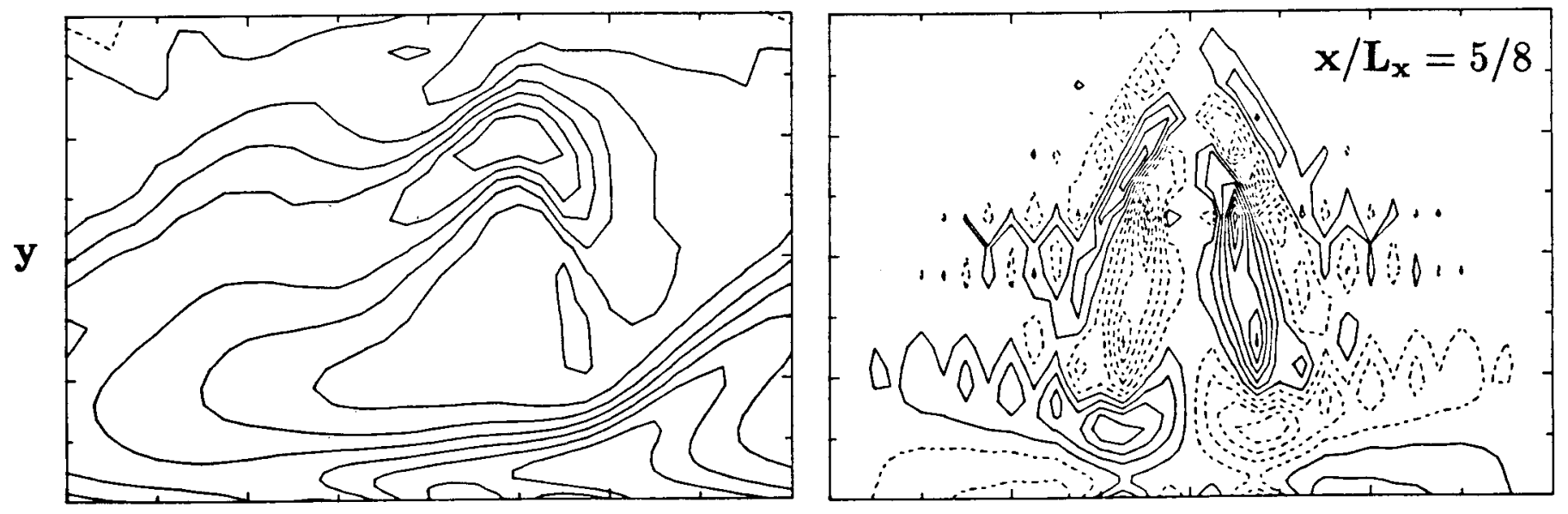

64x64x64 FCBM
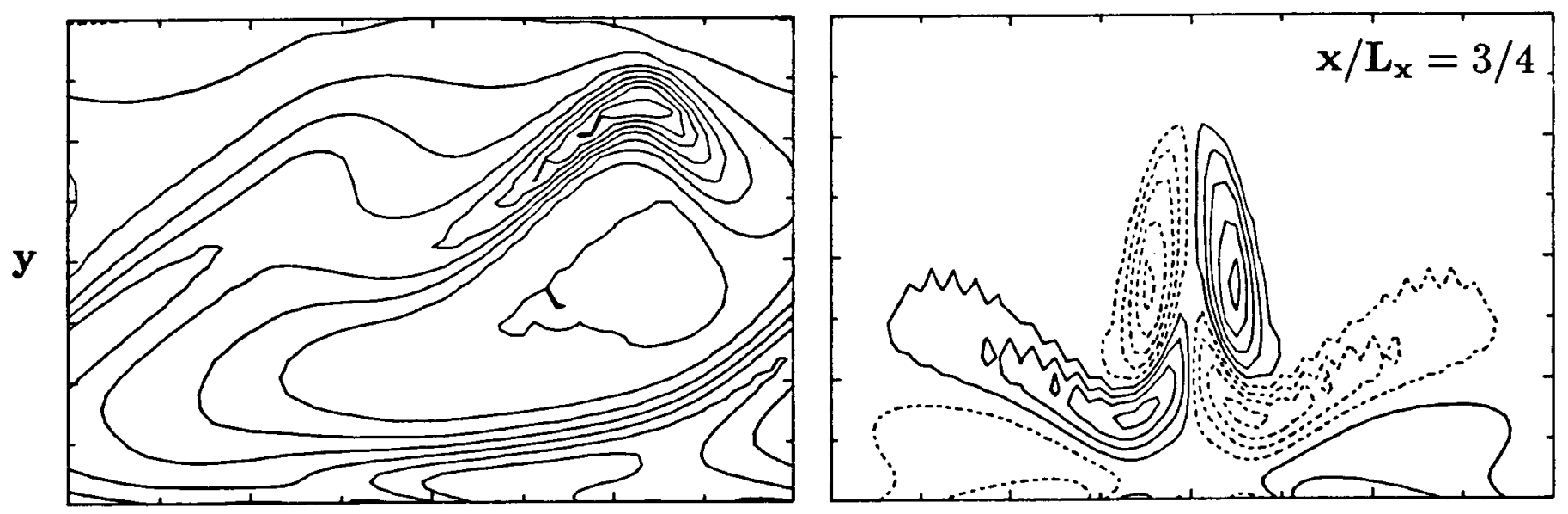

\section{6x128x162 FCBM}
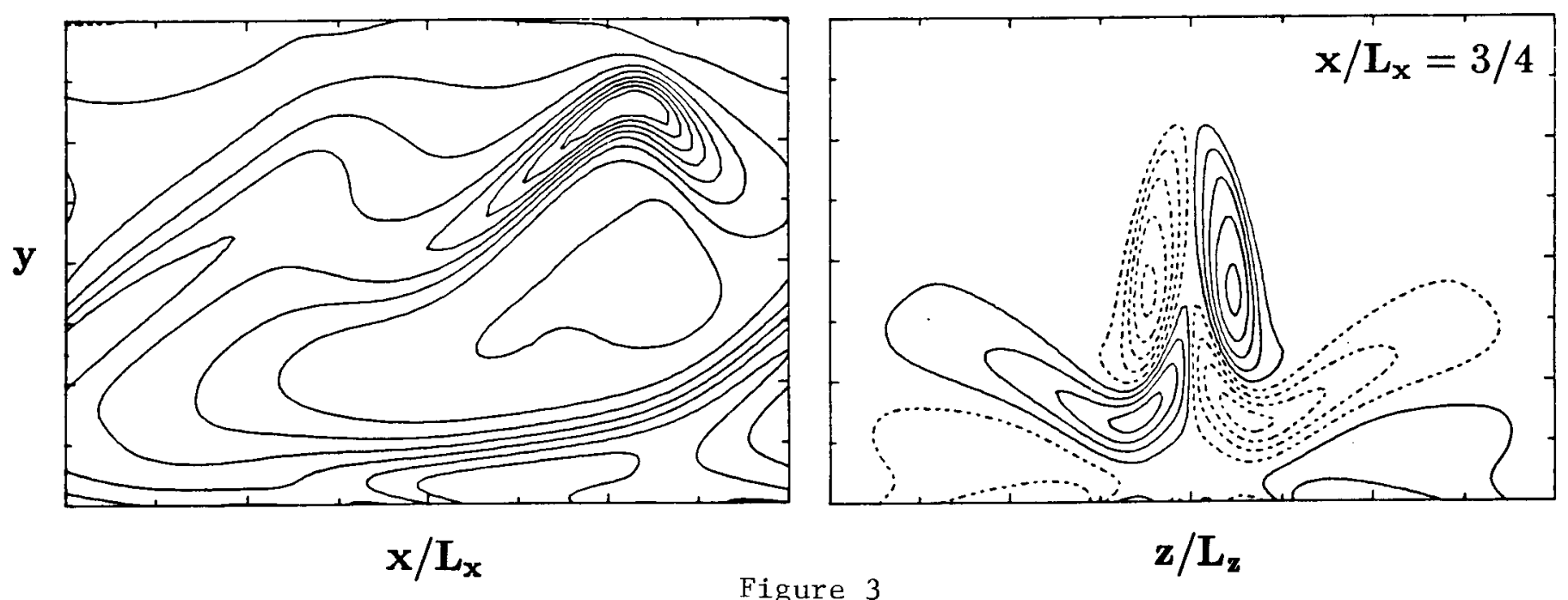

Figure 3 

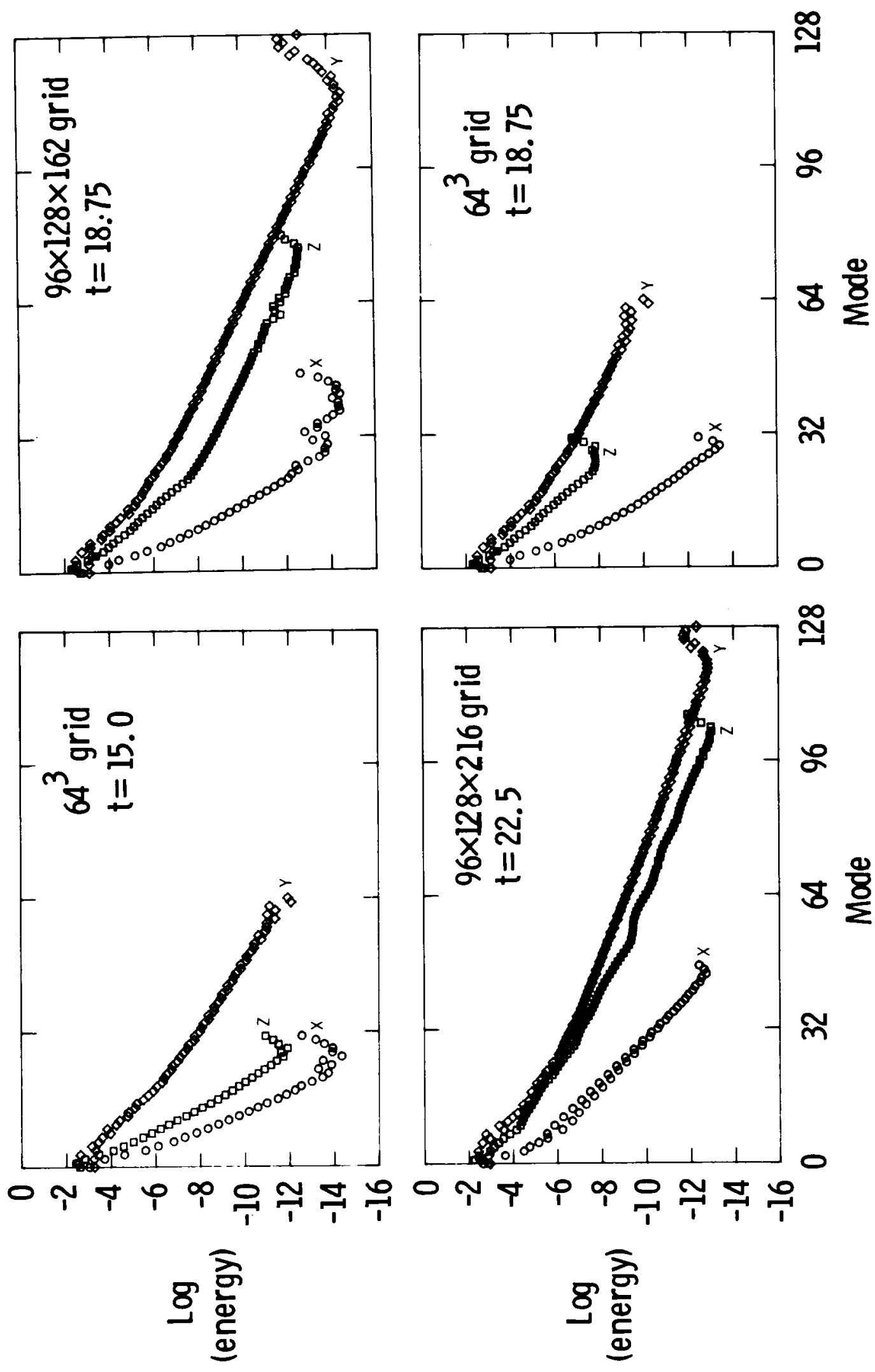

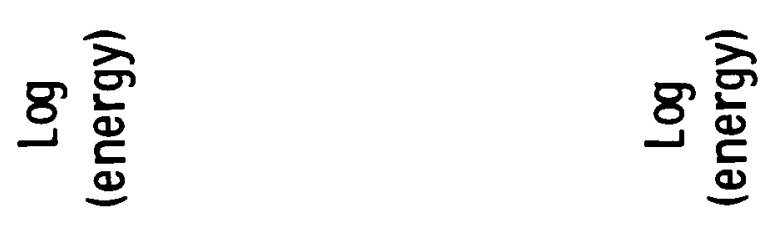



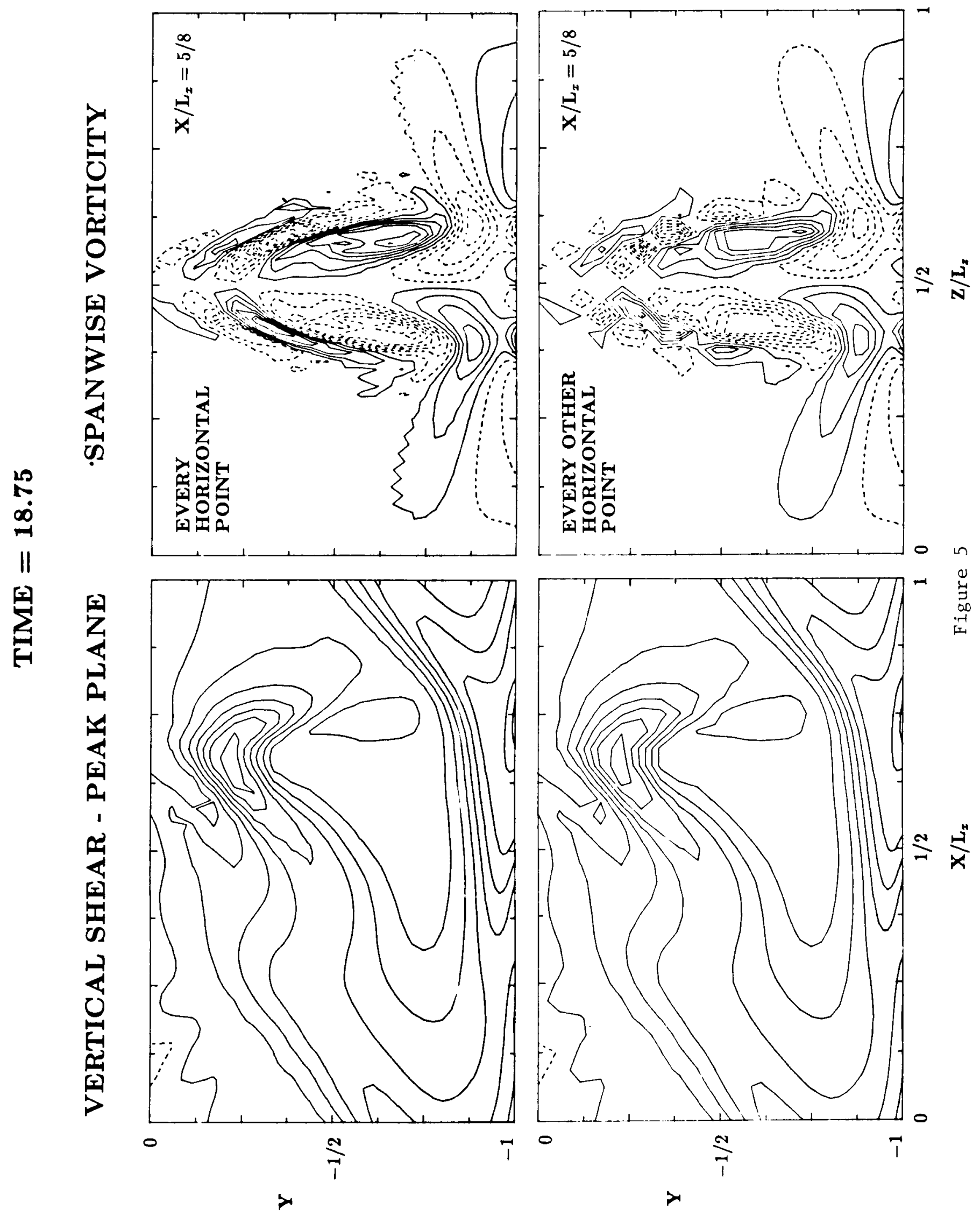


\section{2x50x32 FFDM}
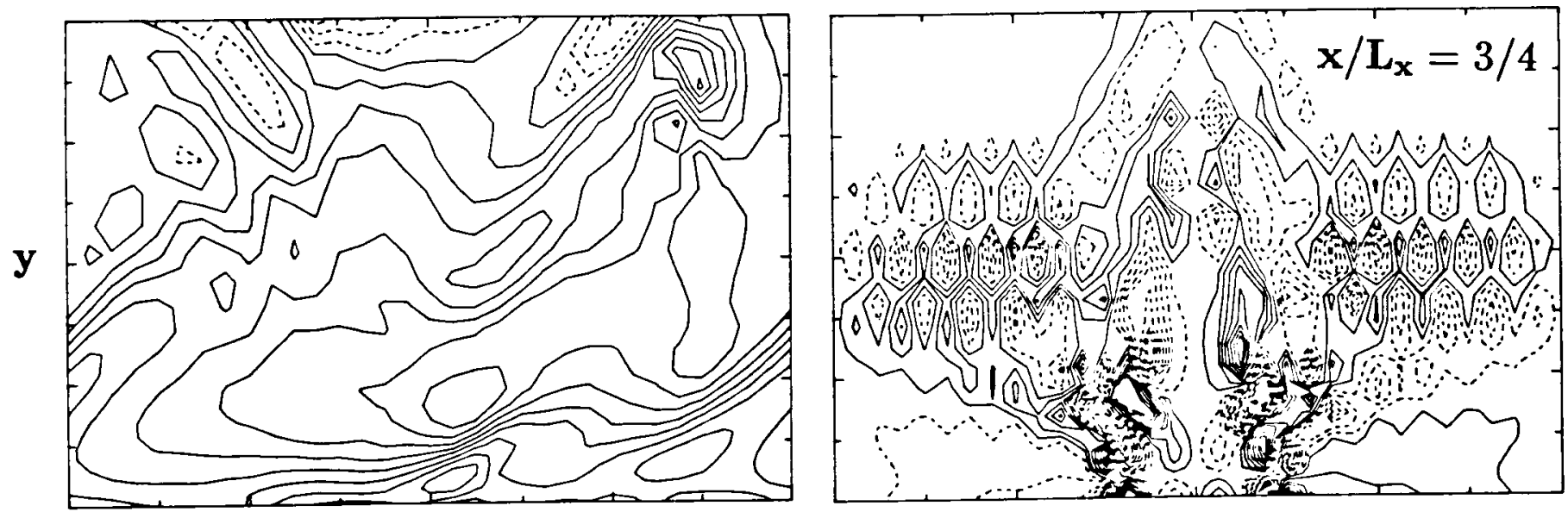

\section{4x64x64 FCBM}
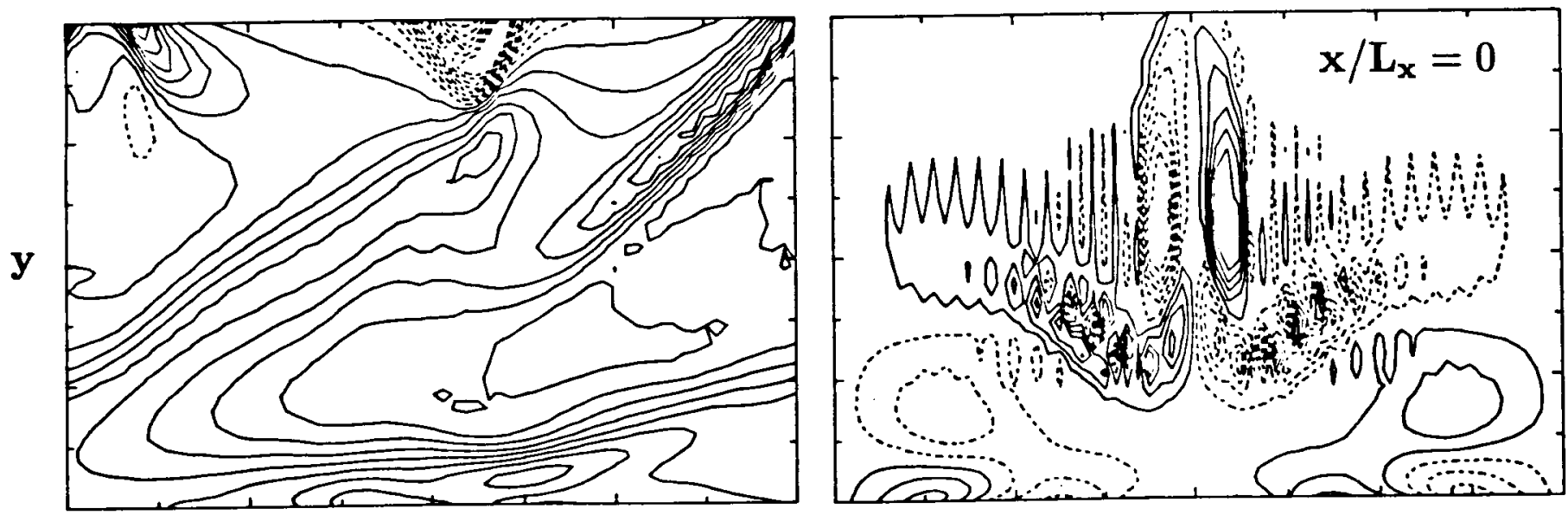

96x128x216 FCBM
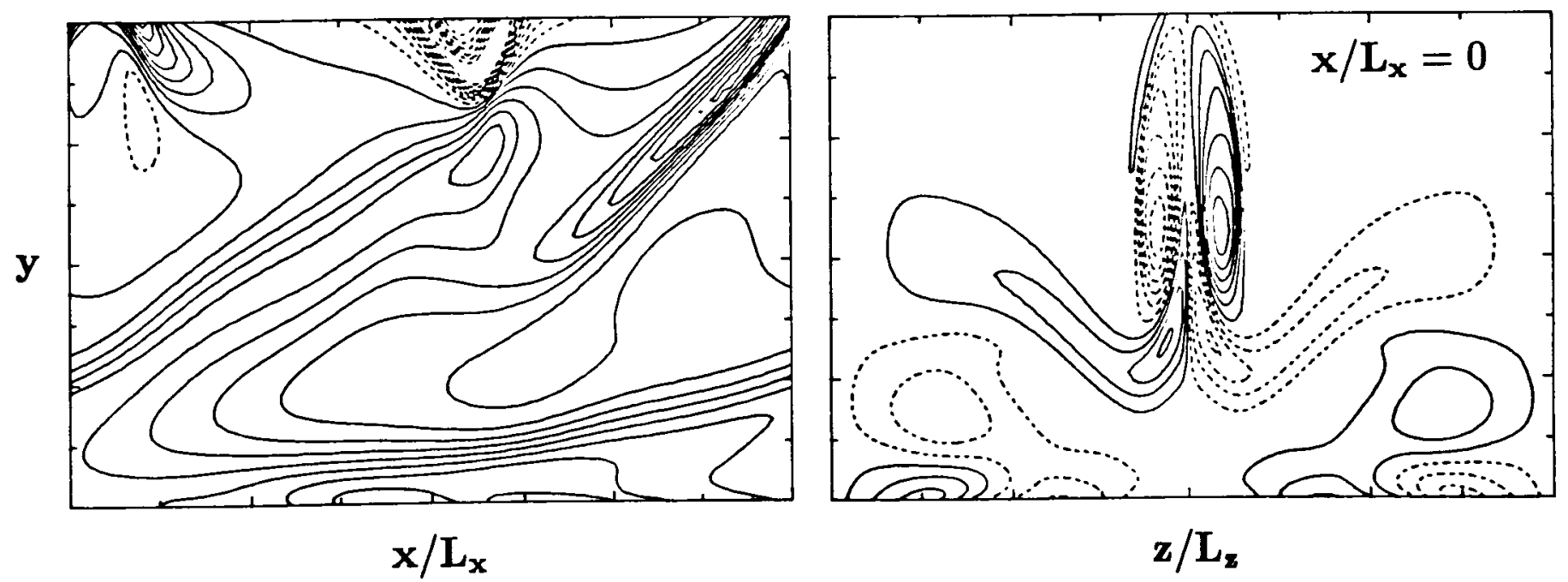

Figure 6 


\section{$32 \times 50 \times 32$ FFDM}
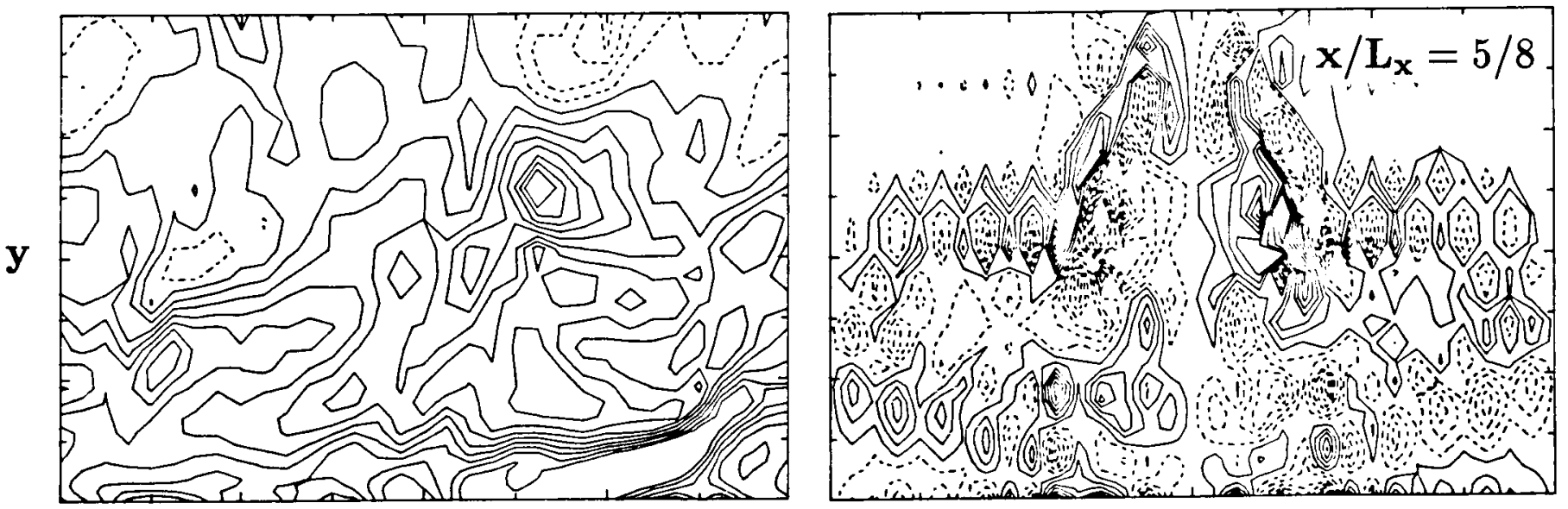

64x64x64 FCBM
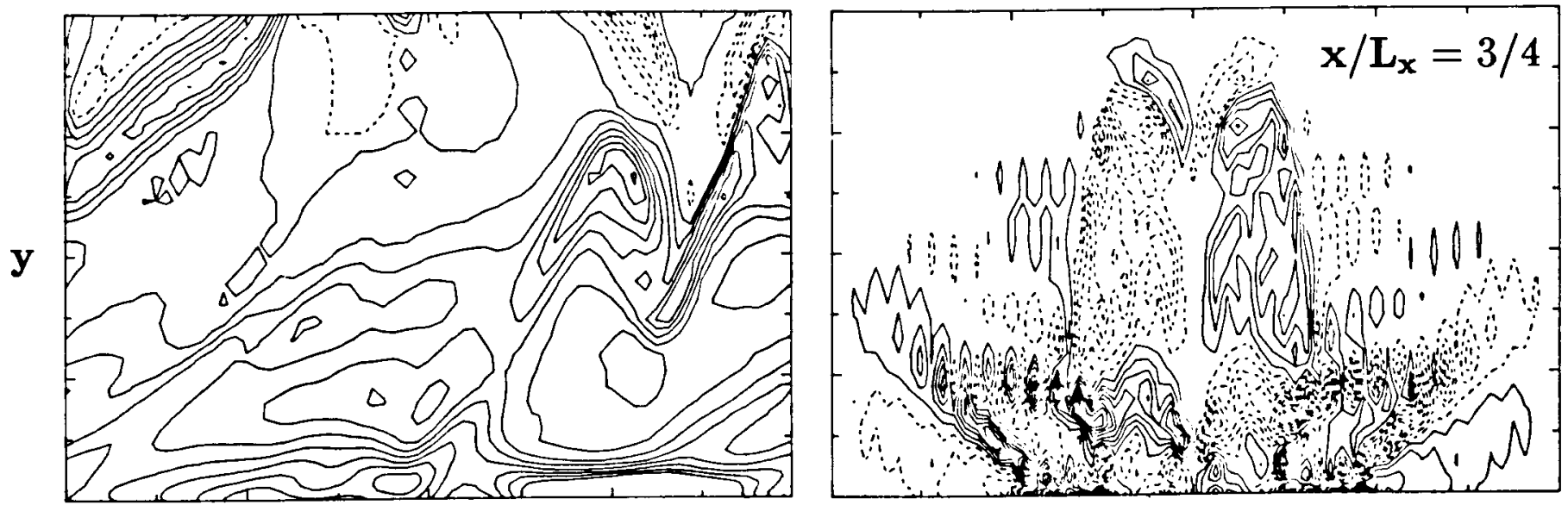

\section{4x128x216 FCBM}
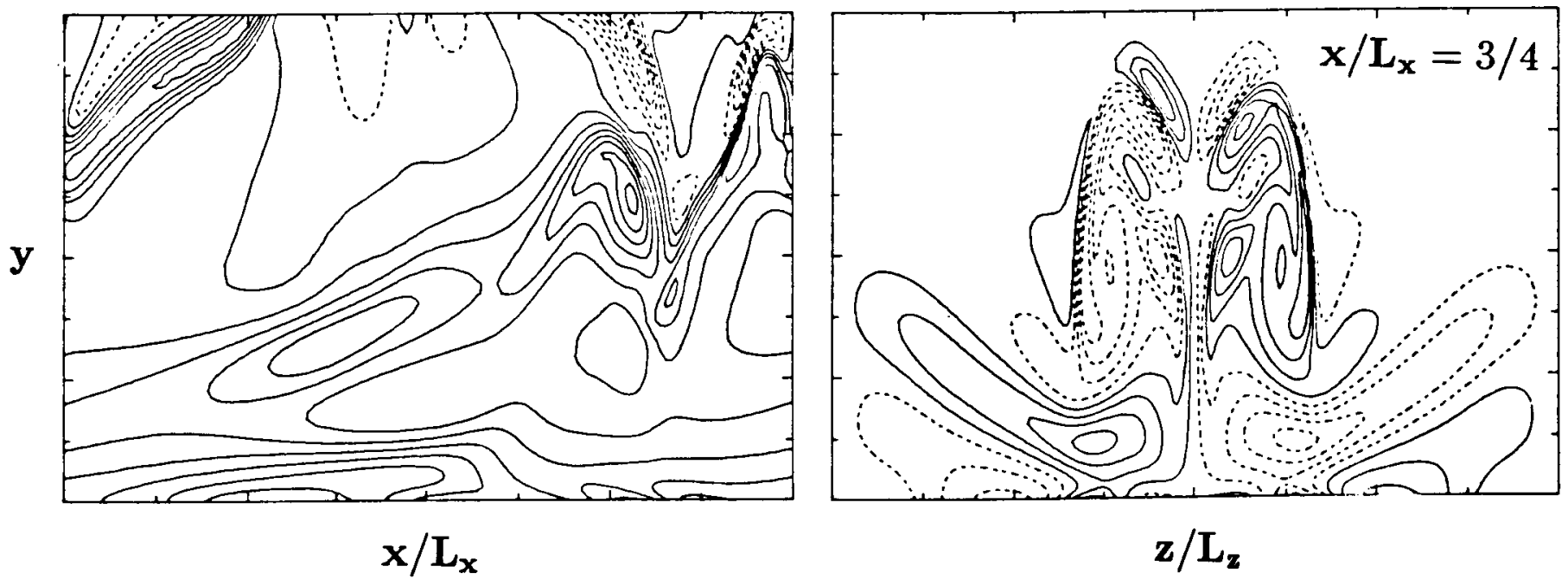

Figure 7 


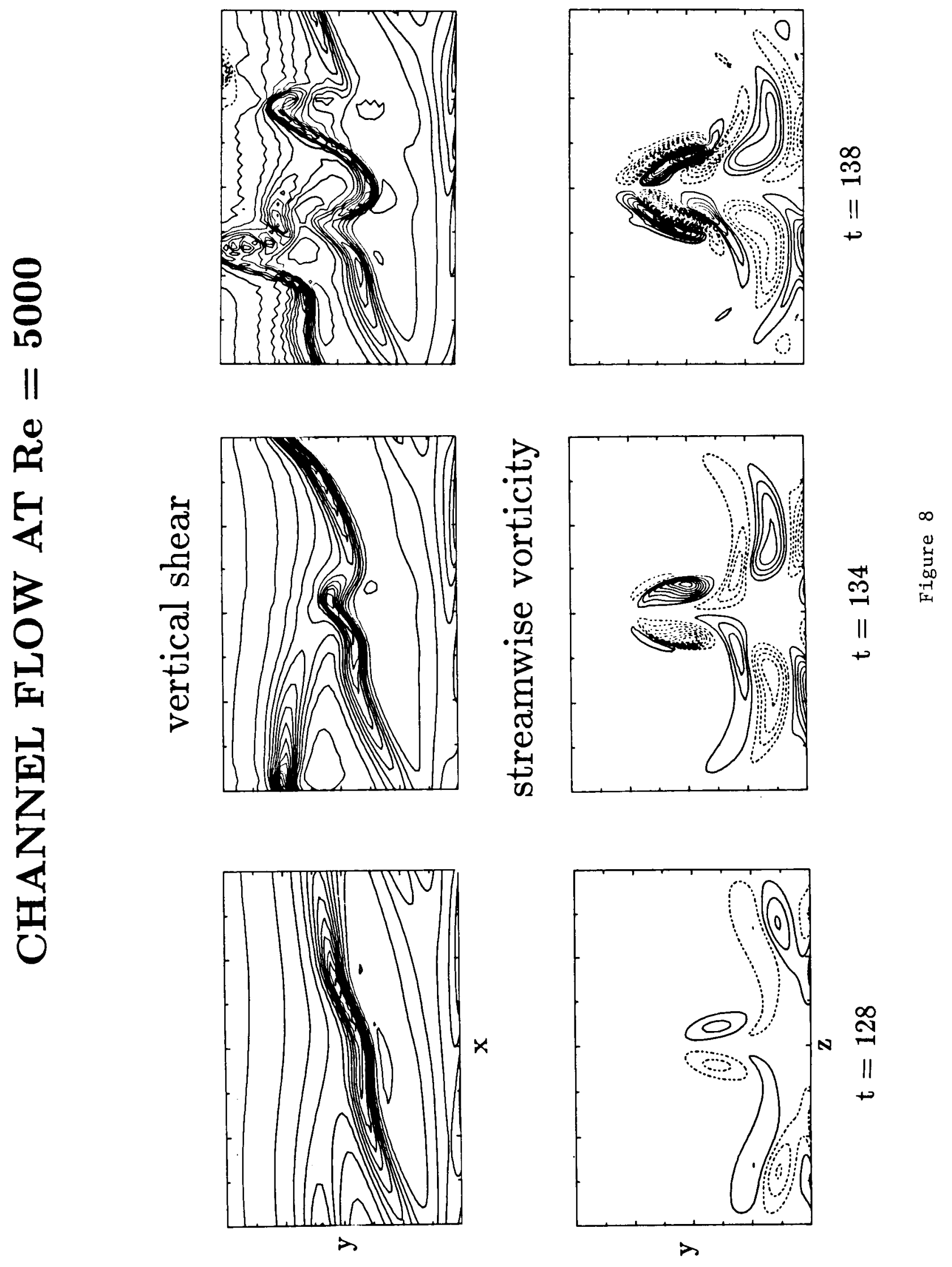



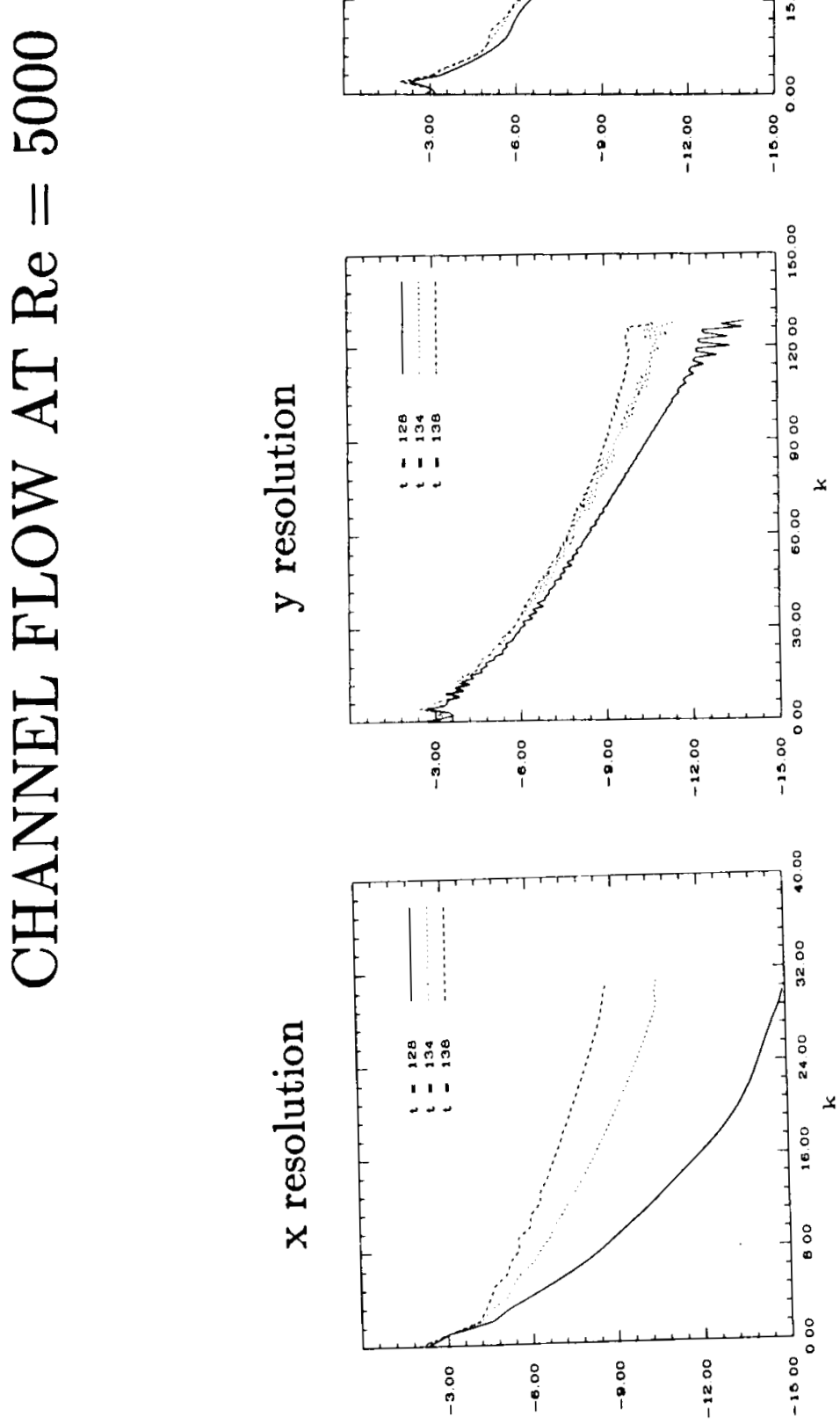

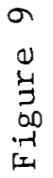




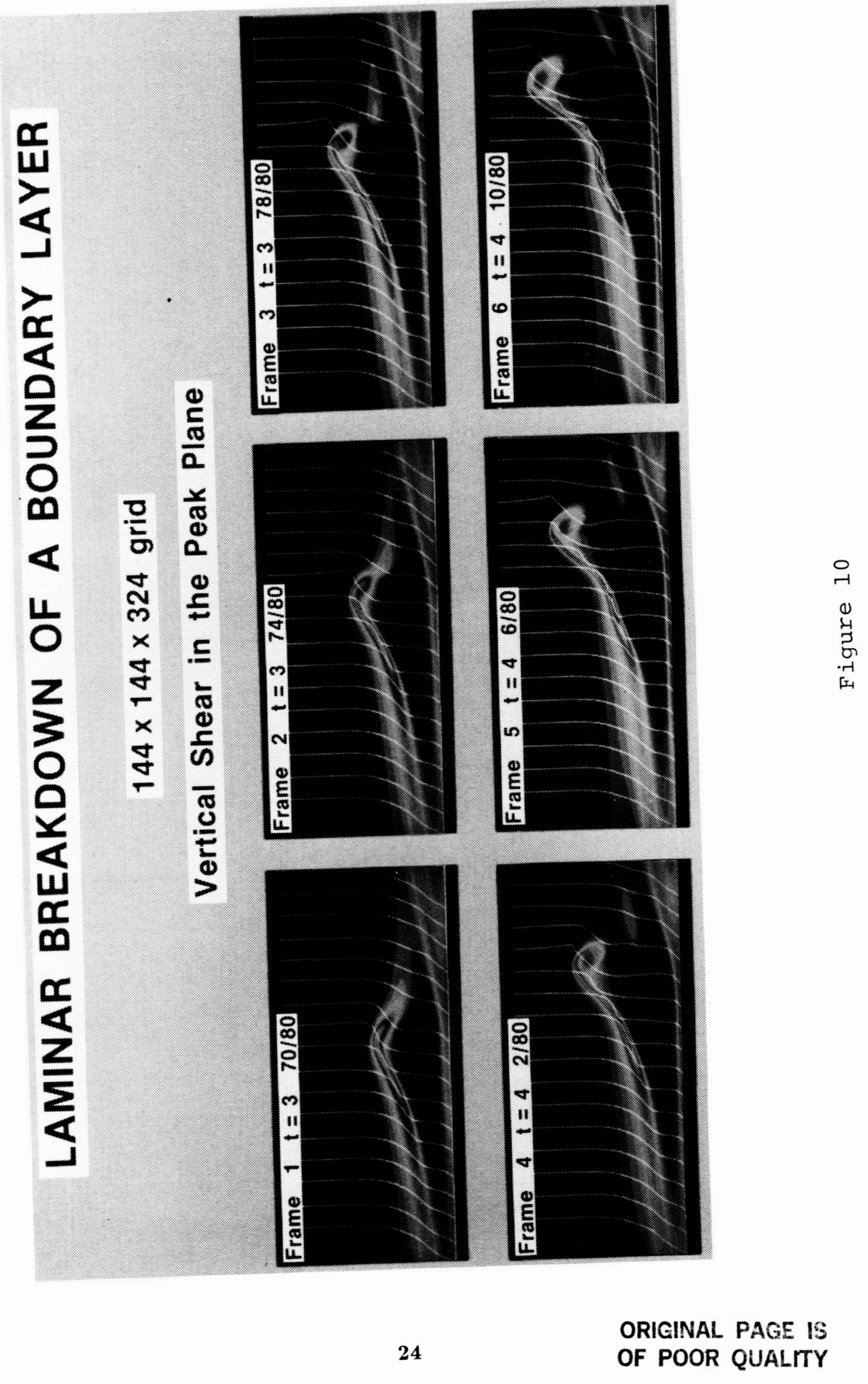


ORIGINAL PAGE IS

OF POOR QUALTY

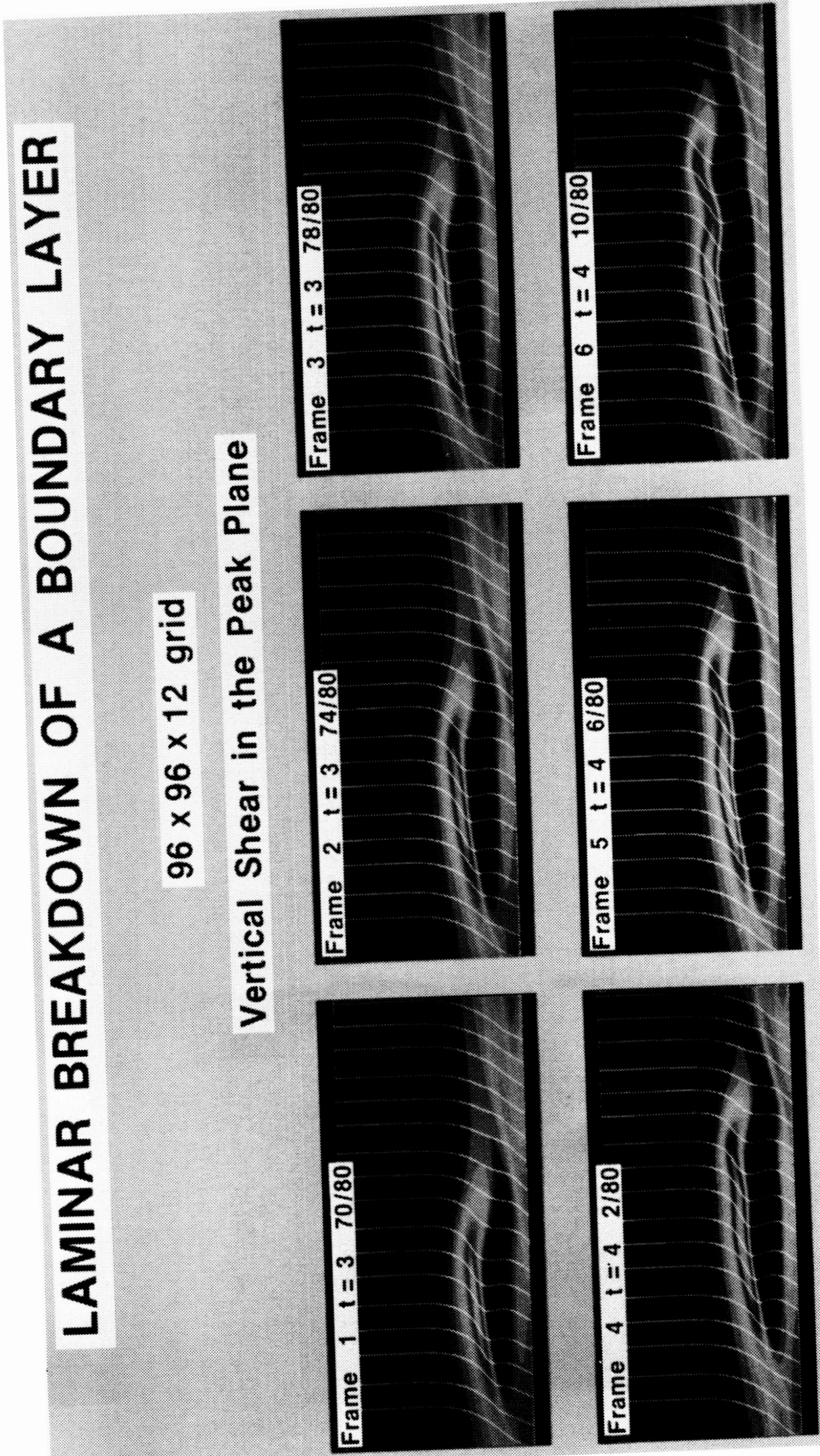

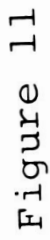




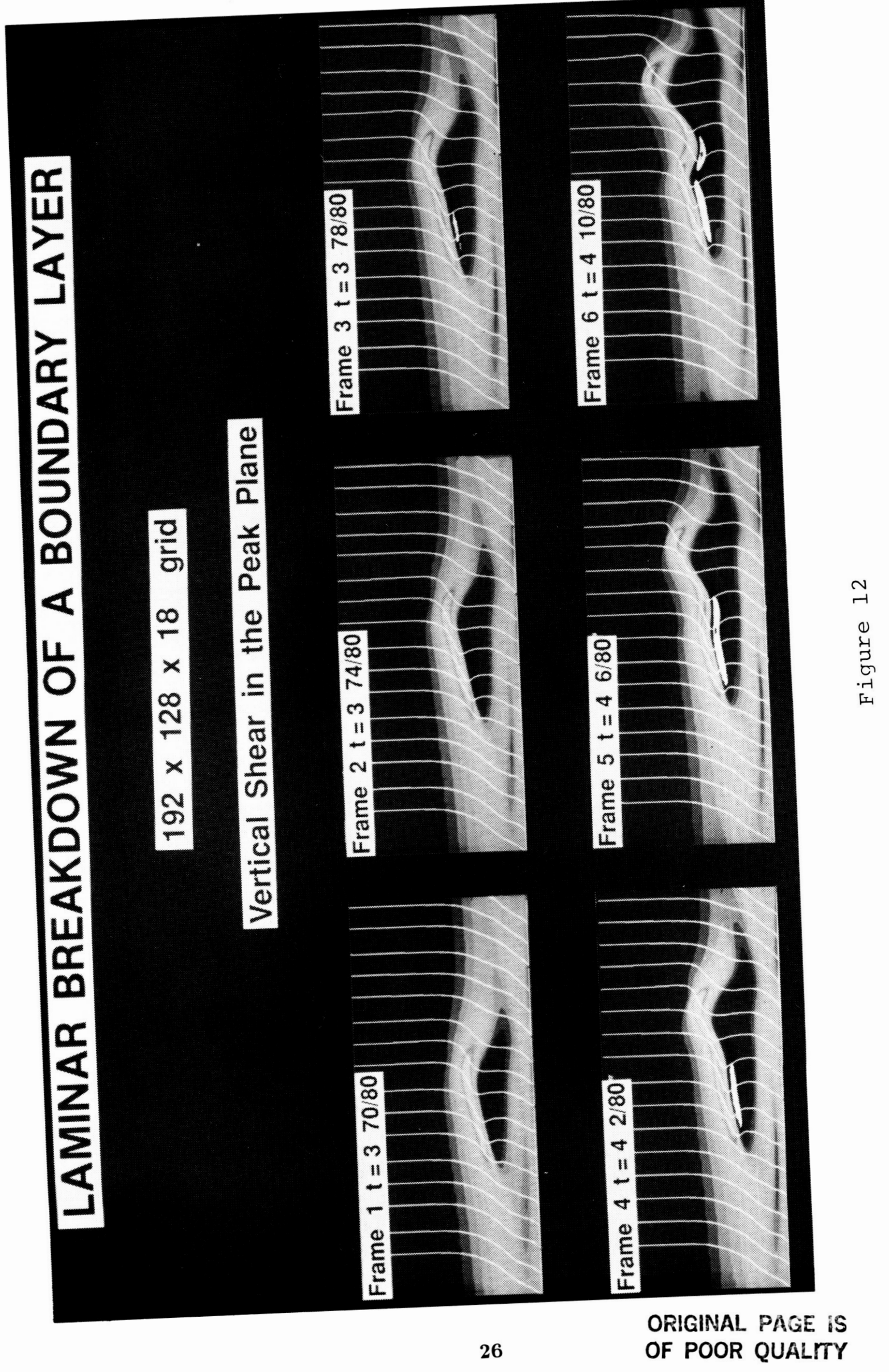


ORIGINAL PAGE IS

OF POOR QUALTTY

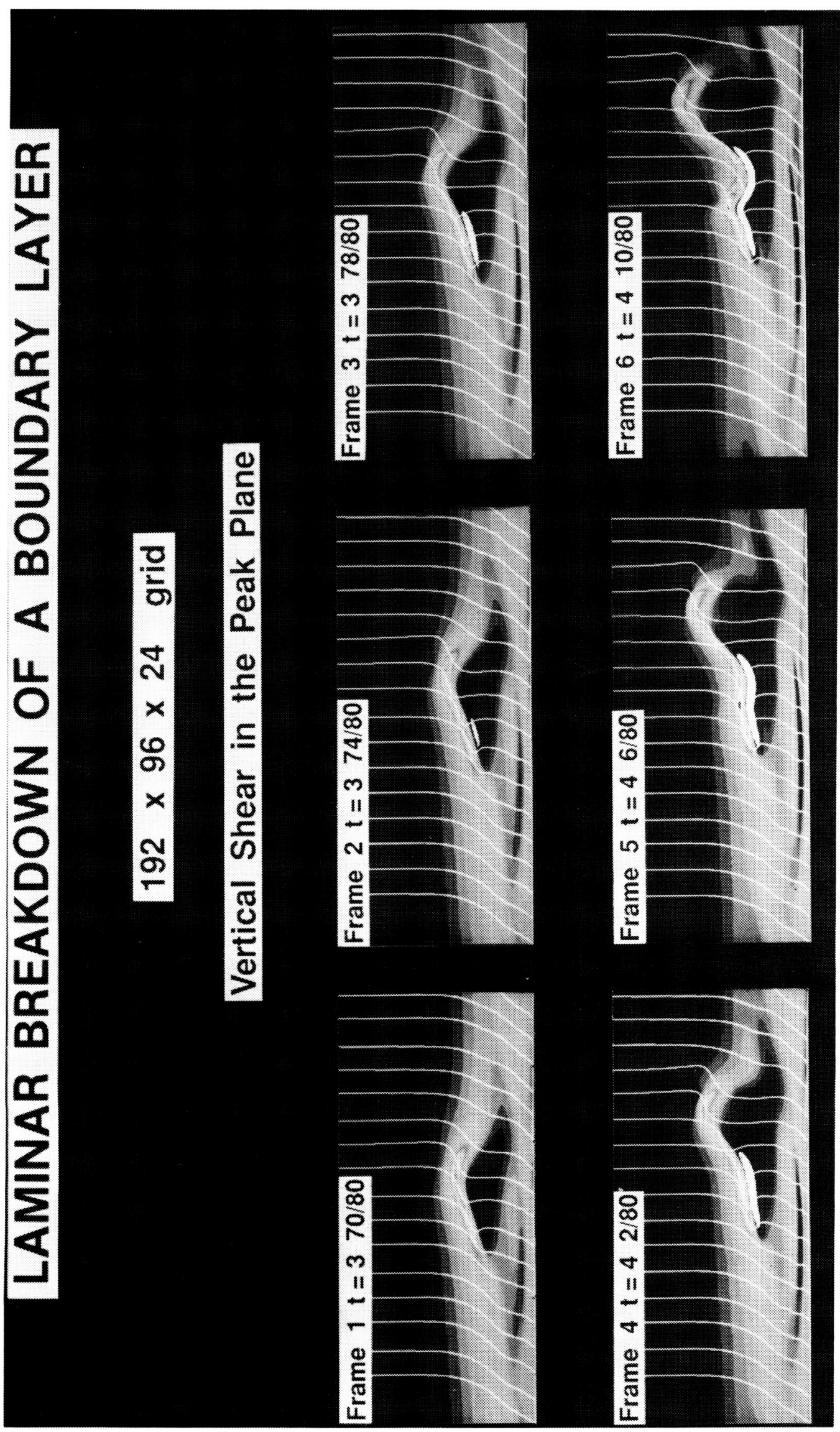

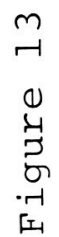




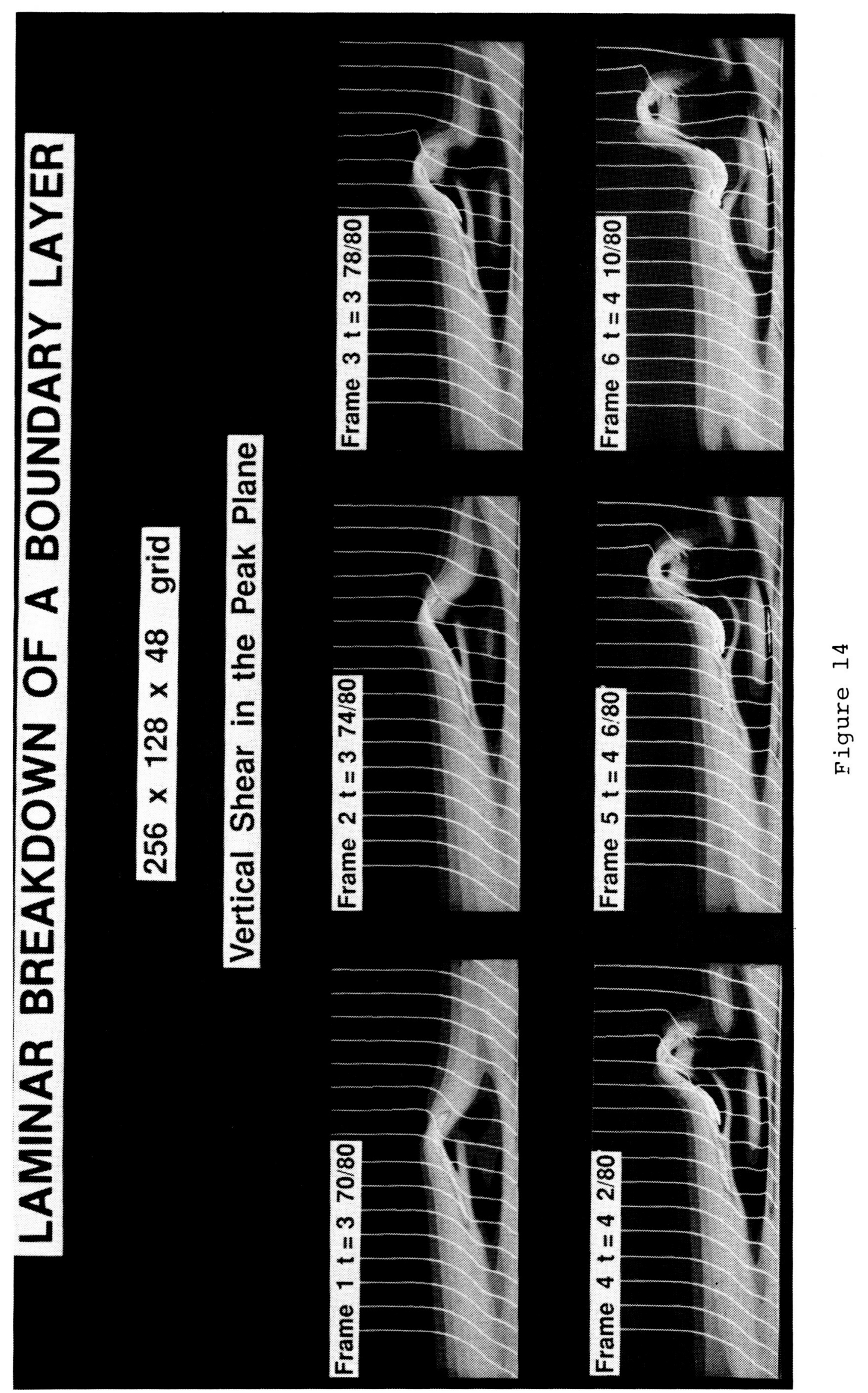




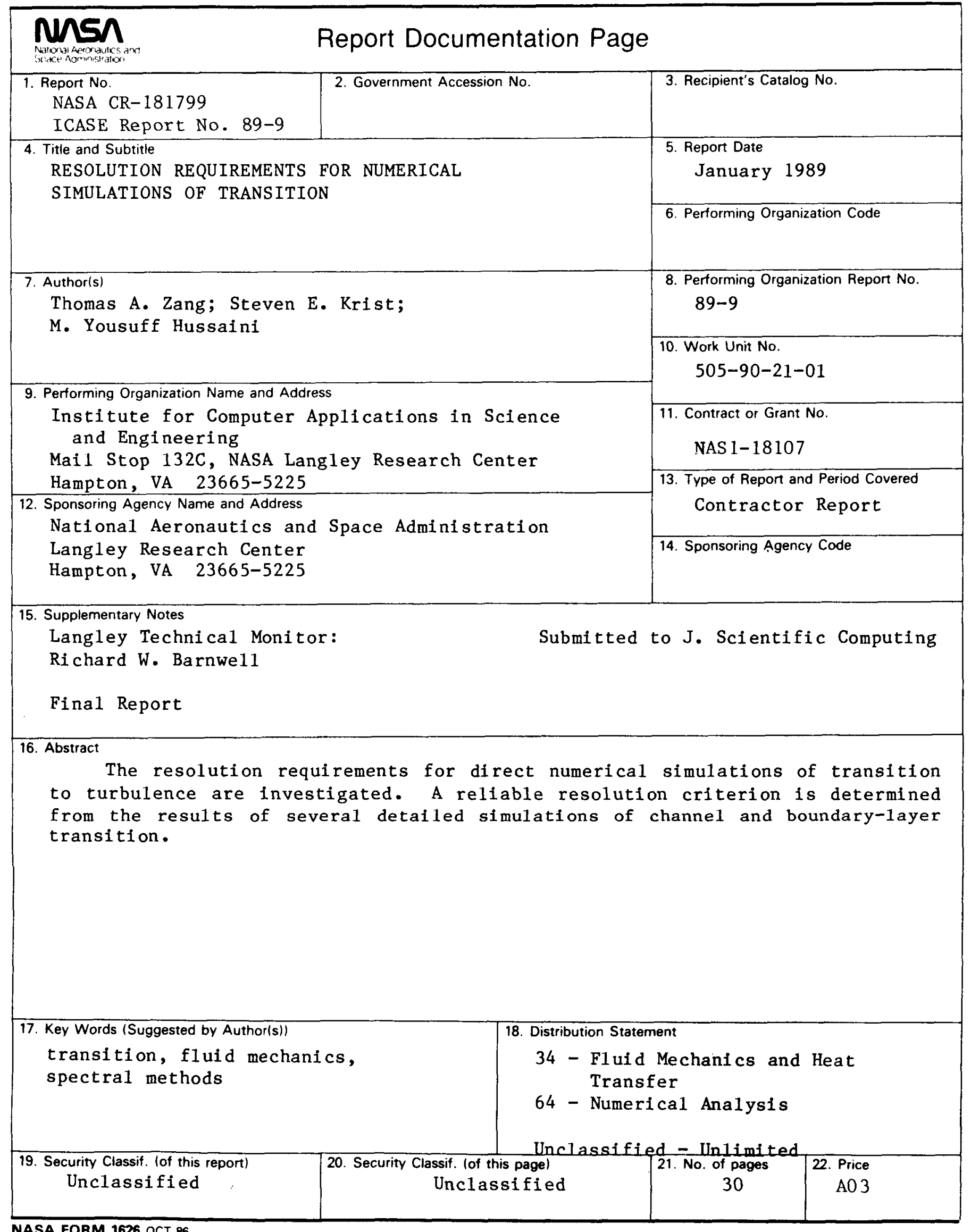

NASA FORM 1626 OCT 86 